\title{
Mechanism Underlying Color Variation in Calla Lily Spathes Based on Transcriptomic Analysis
}

\author{
Ying Fang, Ting Lei, Yanmei Wu, and Xuehua Jin \\ School of Architecture and Urban Planning, Kunming University of Science and Technology, \\ Kunming, Yunnan, China
}

\begin{abstract}
AdDitional INDEX words. anthocyanin, coloration mechanism, procyanidins, transcriptome analysis, Zantedeschia hybrida

ABstract. The calla lily (Zantedeschia hybrida) is a valued ornamental plant due to its unique shape and color variations. To determine the mechanisms responsible for color development in the calla lily spathe, we conducted a comparative transcriptomic analysis of the spathes of the black [Black Girl (B)], pink [Romantic (P)], and white [Ventura (W)] cultivars. The gene expression patterns in six spathe colors, including the preceding three colors as well as the amaranth [Promise (N)], red [Figo (F)], and yellow [Sun Club (Y)] cultivars were analyzed by real-time quantitative polymerase chain reaction (PCR). Transcriptomic analysis identified 25,165 differentially expressed genes. The transcription abundance and expression level of genes annotated as anthocyanidin reductase (ANR1, ANR2), basic-helix-loop-helix (bHLH1), and glutathione S-transferases (GST1) were significantly upregulated in B, and the expression of anthocyanidin synthase (ANS) was highest in B except for $\mathrm{N}$. However, chalcone isomerase (CHI2) and dihydroflavonol 4-reductase (DFR1, DFR2) were expressed at significantly lower levels in $\mathrm{P}, \mathrm{W}$, and $\mathrm{Y}$. Correlation analysis revealed that bHLH1 might act as a positive regulator of $A N S$ expression, promoting anthocyanin synthesis. Moreover, GST1-encoded proteins may be related to the accumulation and transport of both anthocyanin and procyanidin in the calla lily spathe. It is speculated that the formation of the black spathe is related to the accumulation of anthocyanins and procyanidins. However, the low expression of $C H I 2, D F R 1$, and $D F R 2$ may result in the inhibition of anthocyanin synthesis, which may lead to lightening of the spathe color. This preliminary study revealed the mechanism responsible for calla lily spathe color, identifying the key genes involved, thus providing effective gene resources and a theoretical basis for flower color molecular breeding.
\end{abstract}

The flower color of ornamental plants has attracted much attention (Qu et al., 2018). Research on the mechanisms of color formation in nonmodel ornamental plants can lead to a better understanding of plant color-related genetic resources (Tripathi et al., 2018). Flavonoids, carotenoids, and alkaloids provide different coloration to flowers (Hanbali et al., 2013; Kelebek and Selli, 2011). Among these, anthocyanins are one of the most important chromogenic substances in the metabolism of flavonoid compounds in higher plants (Bueno et al., 2012; Tripathi et al., 2018). Their biosynthesis is catalyzed by chalcone synthase (CHS), chalcone isomerase (CHI), flavanone 3-hydroxylase $(\mathrm{F} 3 \mathrm{H})$, flavonoid 3'-hydroxylase $\left(\mathrm{F} 3{ }^{\prime} \mathrm{H}\right)$, dihydroflavonol 4reductase (DFR), ANS, anthocyanidin reductase (ANR), and other enzymes (Grotewold, 2006; Koes et al., 2005; Nguyen et al., 2006; Nováková et al., 2006; Sheng et al., 2018; Tanaka and Ohmiya, 2008). For example, the color variation in Brunfelsia acuminata, Oncidium, and Magnolia sprengeri is a consequence of differences in the expression of genes within the anthocyanin biosynthesis pathway, leading to variations in anthocyanin accumulation (Li et al., 2016; Liu et al., 2012; Shi et al., 2014). In addition, it has been reported that the pigmentation of brown fibers may be due to oxidized-state procyanidins (condensed tannins) and procyanidin (PA) precursors, and high ANR expression leads to greater PA accumulation (Kitamura et al., 2004). At the same time, the development of flower color

Received for publication 15 Apr. 2021. Accepted for publication 19 July 2021. Published online 16 September 2021.

We thank the National Natural Science Foundation of China (No.31760587; No. 31560568) for financial support of this research.

X.H.J. is the corresponding author. E-mail: xh_kimlian@126.com.

This is an open access article distributed under the CC BY-NC-ND license (https://creativecommons.org/licenses/by-nc-nd/4.0/). also depends on the influence of copigmentation, metal ions, and vacuole $\mathrm{pH}$ (Ohmiya, 2010). Differences in the expression of structural genes can lead to variations in anthocyanin accumulation, highlighting the likely roles played by transcription factors in plant color variations. In general, anthocyanin synthesis in plants is regulated by the Myb, bHLH, and WD40 transcription factors, as well as some protein complexes (such as MBW, composed of three transcription factors) that bind to the promoter regions of structural genes (Koes et al., 2005; Lepiniec et al., 2006; Ramsay and Glover, 2005; Saito et al., 2013; Xu et al., 2015). It has been found that anthocyanin transport and accumulation also affect flower color in plants. Anthocyanins are synthesized in the endoplasmic reticulum and are transported to the vacuole by glutathione S-transferases (GST), members of the multidrug and toxic compound extraction (MATE) family, and ATP-binding cassette (ABC) transporters (Francisco et al., 2013; Luo et al., 2018). The transport of PAs by GSTs has also been previously confirmed. For example, it has been found in Arabidopsis (Arabidopsis thaliana), VviGST3, and VviGST4 may play a role in PA transport in the testa (Ricardo et al., 2016).

High-throughput transcriptome sequencing technology has revealed molecular mechanisms in specific biological processes that enable the study of overall gene function and gene structure (Zheng et al., 2015). At present, transcriptome sequencing has been successfully applied to studying the color patterns in many nonmodel plants, greatly improving our understanding of chromogenic genes and their expression patterns (Lin et al., 2018). For example, transcriptomics and differentially expressed gene analysis of the white and purple butterfly orchid (Phalaenopsis amabilis) and the white and pink tea flower (Camellia sinensis) were conducted to obtain candidate genes (structural and regulatory) involved in anthocyanin biosynthesis, revealing the 
mechanisms underlying petal color (Meng et al., 2019; Zhou et al., 2020). Therefore, differences in the abundance of colorrelated genes in anthocyanin biosynthesis pathways of different species may lead to flower color variation.

The calla lily is native to the family Araceae (X. Lei et al., 2017; Singh et al., 1996). It is one of the most popular ornamental plants due to its unique shape and varied colors. Previously, we evaluated the pigment components in the spathe of 27 calla lily cultivars imported from the Netherlands and found that the primary color determinants in the spathe were flavonoids that differed significantly in terms of both total anthocyanin and total flavonol content among the different colors (X. Lei et al., 2017). Previous studies on the calla lily mainly focused on tissue culture, analysis of main chromogenic substances in spathe (X. Lei et al., 2017; Lewis et al., 2003), and genetic diversity (Wei et al., 2016). However, little research has been done on the mechanism of color formation of the spathe.

In this study, transcriptome analysis was carried out on three different-colored calla lily cultivars: black [Black Girl (B)], pink [Romantic (P)], and white [Ventura (W)]. The underlying color mechanism was discussed in combination with the previous studies on the changes in the total anthocyanin content (TAc) (X. Lei et al., 2017). At the same time, qRT-PCR expression analysis was performed on the preceding three cultivars, and three additional colors, including amaranth [Promise (N)], red [Figo (F)], and yellow [Sun Club (Y)]. Finally, the differentially expressed genes (DEGs) that determined spathe color development in the calla lily were screened. This study provides a theoretical basis and reference for the identification, cloning, and functional analysis of the key genes underlying the development of color in calla lily spathes.

\section{Materials and Methods}

Plant materials. Comparative transcriptomic analysis was conducted on six calla lily cultivars (Fig. 1), namely, B, P, W, $\mathrm{Y}, \mathrm{N}$, and $\mathrm{F}$. Transcriptomic sequencing was carried out for the spathes of B, P, and W, and the qRT-PCR expression patterns of

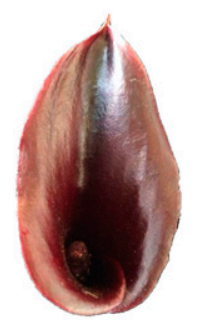

B

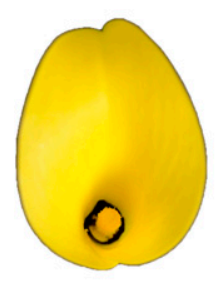

Y

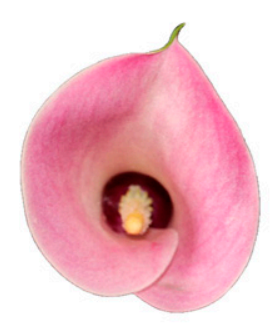

$\mathrm{P}$

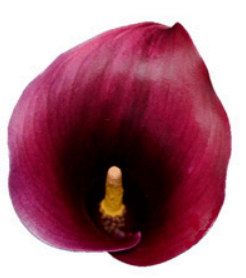

$\mathrm{N}$

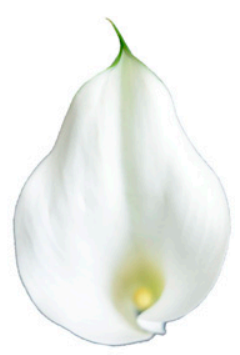

W

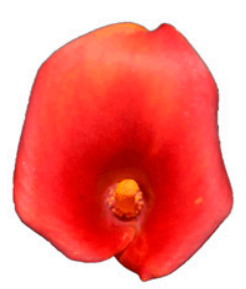

F
Fig. 1. Six calla lily cultivars with different spathe colors: Black Girl (B), Romance (P), Ventura (W), Sun Club (Y), Promise (N), and Figo (F). anthocyanin synthesis-related genes were analyzed for all cultivars. When the spathes were fully open, we collected the test materials at $1500 \mathrm{HR}$. Three individual plants were sampled from each cultivar and $0.2 \mathrm{~g}$ of the uniformly colored part of the spathe was removed for flash-freezing in liquid nitrogen. Samples were stored at $-80^{\circ} \mathrm{C}$ until further use.

RNA EXTRACTION, COMPLEMENTARY DNA LIBRARY CONSTRUCTION, AND TRANSCRIPTOME SEQUENCING. Total RNA was extracted from spathe using an RNA extraction kit (0416-500; Beijing Huayueyang Biological Technology Co., Beijing, China) according to the manufacturer's protocol. The RNA quality was assessed on a bioanalyzer (model 2100; Agilent Technologies, Palo Alto, CA) and confirmed using RNase-free agarose gel electrophoresis (Singh et al., 2015). Following the total RNA extraction, the eukaryotic messenger RNA (mRNA) was enriched using Oligo (dT) (E7530; New England Biolabs, Ipswich, MA) beads, and prokaryotic mRNA was enriched by removing ribosomal RNA using the a Ribo-ZeroTM Magnetic Kit (Epicenter, Madison, WI). The enriched mRNA was then fragmented using fragmentation buffer and reverse-transcribed into complementary DNA (cDNA) with random primers. Second-strand cDNA was synthesized using DNA polymerase I, RNase $\mathrm{H}$, dNTP, and buffer. The cDNA fragments were then purified using a PCR extraction kit (QiaQuick; Qiagen, Venlo, The Netherlands), endrepaired, poly(A) added, and ligated to Illumina (San Diego, CA) sequencing adapters (Fu et al., 2014). The target bands were then size-selected for PCR (Hu et al., 2015), and sequenced using the Illumina HiSeq ${ }^{\mathrm{TM}} 2000$ platform by Gene Denovo Biotechnology Co. (Guangzhou, China). The reads per kilobase per million mapped reads (RPKM) was used to represent the expression levels of different genes.

DE NOVO ASSEMBLY OF TRANSCRIPTOME SEQUENCING DATA AND FUNCTIONAL ANNOTATION. "Single genes" are genes identified by de novo assembly results and the sequence assembly quality is assessed using sequence and base number, length distribution, average coverage, and N50 statistics (Cock et al., 2009). Reads obtained from the sequencing machines include raw reads containing adapters or low-quality bases that will affect the following assembly and analysis. Thus, to get high-quality clean reads, reads were further filtered by fastp [version 0.18.0 (Chen et al., 2018)]. The data were cleaned by removing reads with the following parameters: 1) reads containing adapters; 2) reads containing more than $10 \%$ of unknown nucleotides $(\mathrm{N}) ; 3$ ) low-quality reads containing more than $50 \%$ of low-quality bases [probability that the hypothesis is correct, but rejected = the number of negatives/ the total number, which is a test probability against the sample data $(\mathrm{Q}$-value $) \leq 20)$ ]. The high-quality Illumina reads from three different cDNA libraries of the same cultivar were pooled to produce a calla lily transcriptome database, which was assembled de novo using Trinity software (Grabherr et al., 2011). Transcriptome databases of $\mathrm{B}, \mathrm{P}$, and $\mathrm{W}$ were constructed.

DEG aNALysis and functional enRichment. Single-gene expression was calculated and normalized to RPKM. RNA differential expression analysis was performed by DESeq2 (Love et al., 2014; Robinson et al., 2009) software between two different groups. The genes/transcripts with the parameter of false discovery rate (FDR) $<0.05$ and absolute fold change $(\mathrm{FC}) \geq 2$ were considered as differentially expressed genes/transcripts. The basic annotation of unigenes included protein functional annotation, pathway annotation, clusters of orthologous groups (COG), eukaryotic orthologous groups (KOG) functional annotation, and 
gene ontology (GO) annotation. To annotate the unigenes, we used the BLASTx (Altschul et al., 1990) program with an E-value $<1.0 \times 10^{5}$, the National Center for Biotechnology Information (NCBI) (Nuala et al., 2015) nonredundant protein (Nr) database (Guenter et al., 1998), the SwissProt protein database (Consortium, 2020), the Kyoto Encyclopedia of Genes and Genomes (KEGG) database (Ogata et al., 1999), and the COG/KOG database. Protein functional annotations were then obtained according to the best alignment results (Tatusov et al., 2003). The calculated probability was FDR-adjusted, taking FDR $\leq 0.05$ as a threshold. GO and KEGG terms meeting this condition were defined as significantly enriched in the DEGs. This analysis identified the primary biological functions of the DEGs. This method was further used to explore the mechanism of color change among different cultivars, different plant tissues, plants at different stages of development, or different treatments (Chen et al., 2012a; Jin et al., 2016; Li et al., 2010). Our study investigated the color variation mechanisms of calla lily spathes according to the enrichment pathways and the differential genes in the $\mathrm{B}, \mathrm{P}$, and $\mathrm{W}$ pathways and the significant differences in anthocyanin and flavonol content previously reported by our research group (X. Lei et al., 2017).

QRT-PCR-BASED VERIFICATION AND ANALYSIS. For exploring the highly $\mathrm{F}$ gene expression patterns in different cultivars, the expression of DEGs related to flavonoid biosynthetic and transcriptional pathways was tested using qRT-PCR. Nineteen unigenes involved in the anthocyanin metabolism pathway were selected for qRT-PCR analysis to verify the expression results determined by RNA-sequencing. All analyses were done in triplicate on three biological replicates, and the specific primers are listed in Supplemental Table 1. qRT-PCR was performed using SYBR Premix Ex Taq Perfect Real Time (TaKaRa, Tokyo, Japan) and using a sequence detector (Applied Biosystems ABI Prism 7500; Thermo Fisher Scientific, Waltham, MA). The expression patterns of key genes in the anthocyanin metabolism pathway in the spathe were verified. qRT-PCR was performed using the following conditions: pre-denaturation at $95^{\circ} \mathrm{C}$ for $10 \mathrm{~min}$, denaturation at $95^{\circ} \mathrm{C}$ for $15 \mathrm{~s}$, annealing at the optimum temperature for $30 \mathrm{~s}$, extension at $60^{\circ} \mathrm{C}$ for $30 \mathrm{~s}$, for 40 cycles. The relative gene expression levels were calculated using the delta-delta $\mathrm{Ct}$ method $\left(2^{-\Delta \Delta \mathrm{ct}}\right.$ ) (Livak and Schmittgen, 2001), with Cm-Actin and $\beta$-Tubulin expression used as internal standards (repeated in triplicate). Finally, to better analyze the relationship between key genes and total anthocyanin, SPSS software (IBM SPSS Statistics version 24.0; IBM Corp, Armonk, NY) was used to calculate the correlation between the expression levels of key genes and total anthocyanin content, which is the Pearson correlation coefficient $\left(R^{2}\right)$. The closer $R^{2}$ is to 1 , the stronger is the correlation.

\section{Results}

SEQUENCING AND DE NOVO ASSEMbly OF TRANSCRIPTOME DATA. To further understand the underlying molecular mechanisms of spathe color in the calla lily, three libraries representing B, P, and $\mathrm{W}$ were constructed. After trimming the adapters and filtering out low-quality reads $(\mathrm{N} \%>10 \%), 73,510,006$ reads from $\mathrm{B}, 41,661,782$ reads from $\mathrm{P}$, and $37,623,484$ reads from $\mathrm{W}$ were collected. The GC content was $50.5 \%, 50.55 \%$, and $51.05 \%$ for $\mathrm{B}, \mathrm{P}$, and $\mathrm{W}$, respectively, and the $\mathrm{Q} 20$ ratio (test error $<1 \%$ ) was $96.60 \%, 96.86 \%$, and $97.83 \%$, respectively. The sequencing results showed that the GC content was $\approx 50.5 \%$ (Table 1 ), and the unknown base sequence fragment $\mathrm{N}$ of the three cultivars
Table 1. Transcriptome sequencing original read and assembly data for calla lily cultivars.

\begin{tabular}{lccc}
\hline Cultivar & No. & Q20 (\%) $)^{\mathrm{z}}$ & GC (\%) \\
\hline Black Girl & $73,510,006$ & 96.6 & 50.51 \\
Romantic & $41,661,782$ & 96.86 & 50.55 \\
Ventura & $37,623,484$ & 97.83 & 51.05 \\
\hline
\end{tabular}

${ }^{\mathrm{z}}$ Proportion of guanine and cytosine to all bases in nucleic acid.

${ }^{\mathrm{y}}$ Quality control value of sequencing.

analyzed by transcriptome was 0 in all cases, indicating that the sequencing quality was high and further analysis could be carried out. The high-quality reads in the transcriptome database contained a total of 85,907 sequences, with an average transcript length of 785 base pairs (bp), among which the longest sequence was 15,804 bp and the shortest was $201 \mathrm{bp}$ (Table 2, Fig. 2). Among these, $95.5 \%$ of the sequences were within 200 to 3000 bp (Fig. 2).

FunCtional ANNOTATION AND CLASSIFICATION. For an overall understanding of all unigene sequence-function information, the assembled transcriptome data were compared and annotated in the Nr, SwissProt, KEGG, KOG, and GO databases. Approximately 85,907 assembled unigenes were functionally annotated using BLASTx against the NCBI Nr, SwissProt, Protein family (Pfam), KOG, GO, and KEGG databases. From this, 29,816 (34.7\%) transcripts were annotated to $\mathrm{Nr}, 20,897(24.3 \%)$ to SwissProt, $10,322(12 \%)$ to KEGG, and $18,034(20.1 \%)$ to KOG (Fig. 3). Because of the lack of a complete calla lily genome sequence, the unigenes showed top matches to Elaeis guineensis at $24.4 \%$ similarity, followed by Phoenix dactylifera (20.5\%), and Nelumbo nucifera (12.8\%) (Fig. 4).

GO FUNCTIONAL ANNOTATION AND KEGG PATHWAY ENRICHMENT OF DEGs. To identify candidate genes related to anthocyanin biosynthesis, B, P, and W DEGs in calla lily spathes were analyzed by pairwise comparisons ( $\mathrm{P}$ vs. $\mathrm{B}, \mathrm{W}$ vs. $\mathrm{B}, \mathrm{W}$ vs. $\mathrm{P}$ ). A total of 25,165 DEGs were identified in the calla lily library, including 10,130 upregulated genes and 15,035 downregulated genes. There were 8880 significantly DEGs in P vs. B, including 3465 upregulated and 5235 downregulated genes. In W vs. P, 8395 genes were significantly differentially expressed, including 6162 that were upregulated and 2233 that were downregulated. In W vs. B, 7890 genes were significantly differentially expressed, including 5228 upregulated and 2662 downregulated genes (Fig. 5).

We annotated the GO function of DEGs obtained by comparison (Fig. 6). GO functional annotation indicated that DEGs were divided into 45 functional groups within biological processes, cellular components, and molecular functions. In the biological process category, most DEGs were classified as metabolic processes $(10,440)$ and cellular processes $(9759)$, followed by organic metabolic processes (8086). In the cell component category, cell (7462) and cell part (7461) were relatively dominant, followed by organelles (7147) and membrane group (7083). Among the molecular functions, catalytic activity (9448) and binding activity (9252) were the most representative.

Table 2. Assembly of sequencing results in calla lily.

\begin{tabular}{ccccc}
\hline & $\begin{array}{c}\text { Max } \\
\text { length (bp) }\end{array}$ & $\begin{array}{c}\text { Max } \\
\text { length (bp) }\end{array}$ & $\begin{array}{c}\text { Avg } \\
\text { length (bp) }\end{array}$ & N50 (bp) \\
\hline 85,907 & 15,804 & 201 & 785 & 1,557 \\
\hline${ }^{\mathrm{z}}$ Quality of genome splicing. bp = base pair.
\end{tabular}




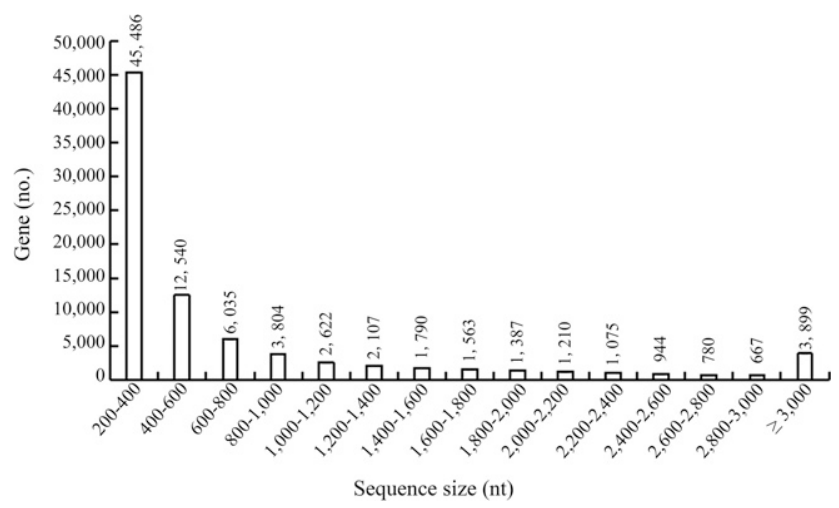

Fig. 2. Assembled unigene length distribution of calla lily.

Although the comparison of the three cultivars showed similar GO functional classification patterns, they differed in enrichment classification (Supplemental Table 2). GO enrichment analysis showed that genes associated with the design and color of phenylalanine and flavonoid biosynthesis were significantly enriched. In P vs. B, 12, 11, and 2 unigenes were annotated to the phenylalanine biosynthesis pathway, flavonoid biosynthesis pathway, and flavonoid flavonol biosynthetic pathway, respectively. In $\mathrm{W}$ vs. $\mathrm{B}$ and $\mathrm{W}$ vs. $\mathrm{P}$, there were 12 and 10 significantly enriched unigenes annotated as involved in phenylalanine biosynthesis pathways, 8 and 13 unigenes in flavonoid biosynthesis pathways, and 1 unigene potentially involved in the flavonol biosynthesis pathway, respectively.

The identification of DEGs in the anthocyanin biosynthesis pathway related to different-colored spathes can provide important information for the mechanism of color development in the calla lily. The primary methods of color development in the spathes of the three cultivars were further investigated by KEGG pathway analysis. It was found that the DEGs were divided into the following 10 categories and 34 KEGG terms: amino acid metabolism, biosynthesis of other secondary metabolites, carbohydrate metabolism, energy metabolism, environmental adaptation, global and overview, lipid metabolism, metabolism of cofactors and vitamins, metabolism of other amino acids, metabolism of terpenoids and polyketides, and signal transduction (Fig. 7). Comparing the

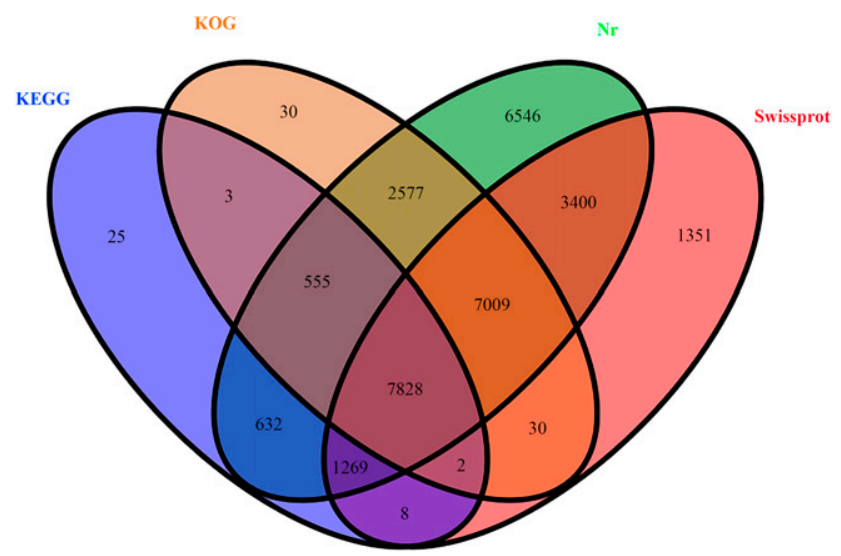

Fig. 3. Venn diagram of unigene annotation using nucleotide sequence database $(\mathrm{Nr})$, clusters of orthologous groups for eukaryotic complete genomes (KOG), Kyoto Encyclopedia of Genes and Genomes (KEGG), and SwissProt protein sequence database (SwissProt) databases.

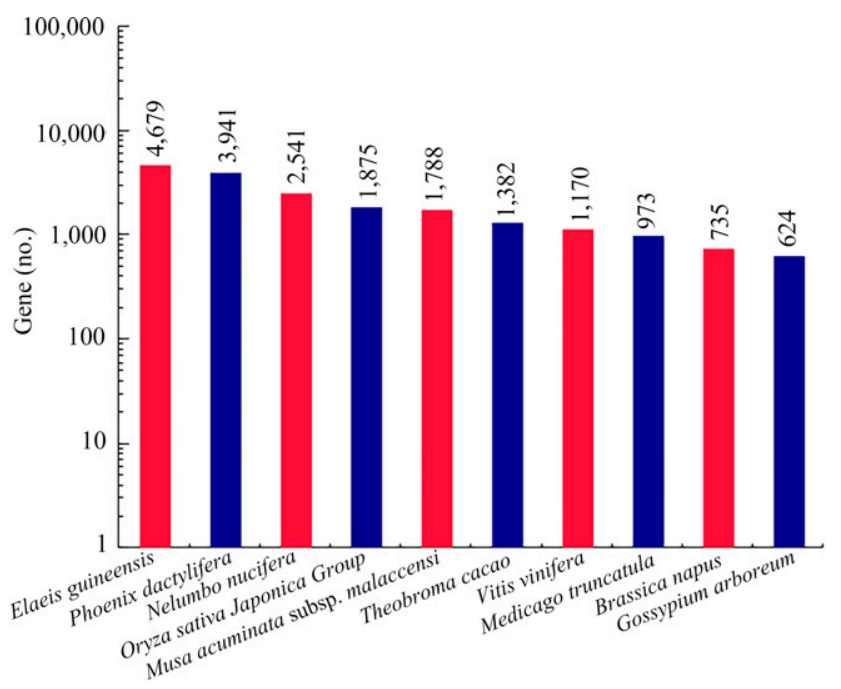

Fig. 4. Species taxonomy based on the nucleotide sequence database ( $\mathrm{Nr}$ ) database.

three cultivars, most DEGs were found in the carbohydrate metabolism category, followed by global and overview. We were particularly interested in this pathway and in identifying the specific genes involved, because the color of the spathe is influenced by the expression of numerous genes involved in the anthocyanin biosynthesis pathway, leading to differences in the type and content of anthocyanin. Therefore, enrichment analysis of DEGs mapped to KEGG biopathways was carried out to clarify the enrichment in biological function of each comparison group of DEGs (Supplemental Table 3). The identified DEGs were mapped to 12 genes in the "biosynthesis of other secondary metabolites" pathway, four of which are related to the flower color formation process. The flavonoid biosynthesis pathway contained the most significant DEG enrichment, followed by the phenylalanine biosynthesis pathway. Specifically, there were 843 DEGs annotated to 119 KEGG metabolic pathways in P vs. B. Among them, 35 DEGs were annotated to the phenylalanine biosynthesis pathway (with 28 upregulated and seven downregulated), and 13 DEGs were annotated to the flavonoid biosynthesis pathway (nine upregulated and four downregulated), which were significantly enriched. Three DEGs were related to flavonol biosynthesis (all downregulated), and one DEG was related to anthocyanin

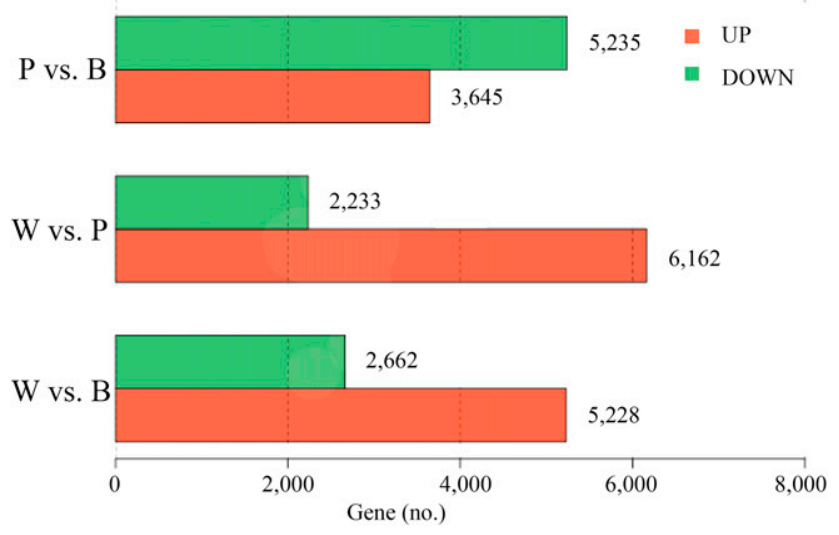

Fig. 5. Analysis of global gene expression among three calla lily cultivars: Black Girl (B), Romance (P), and Ventura (W). 


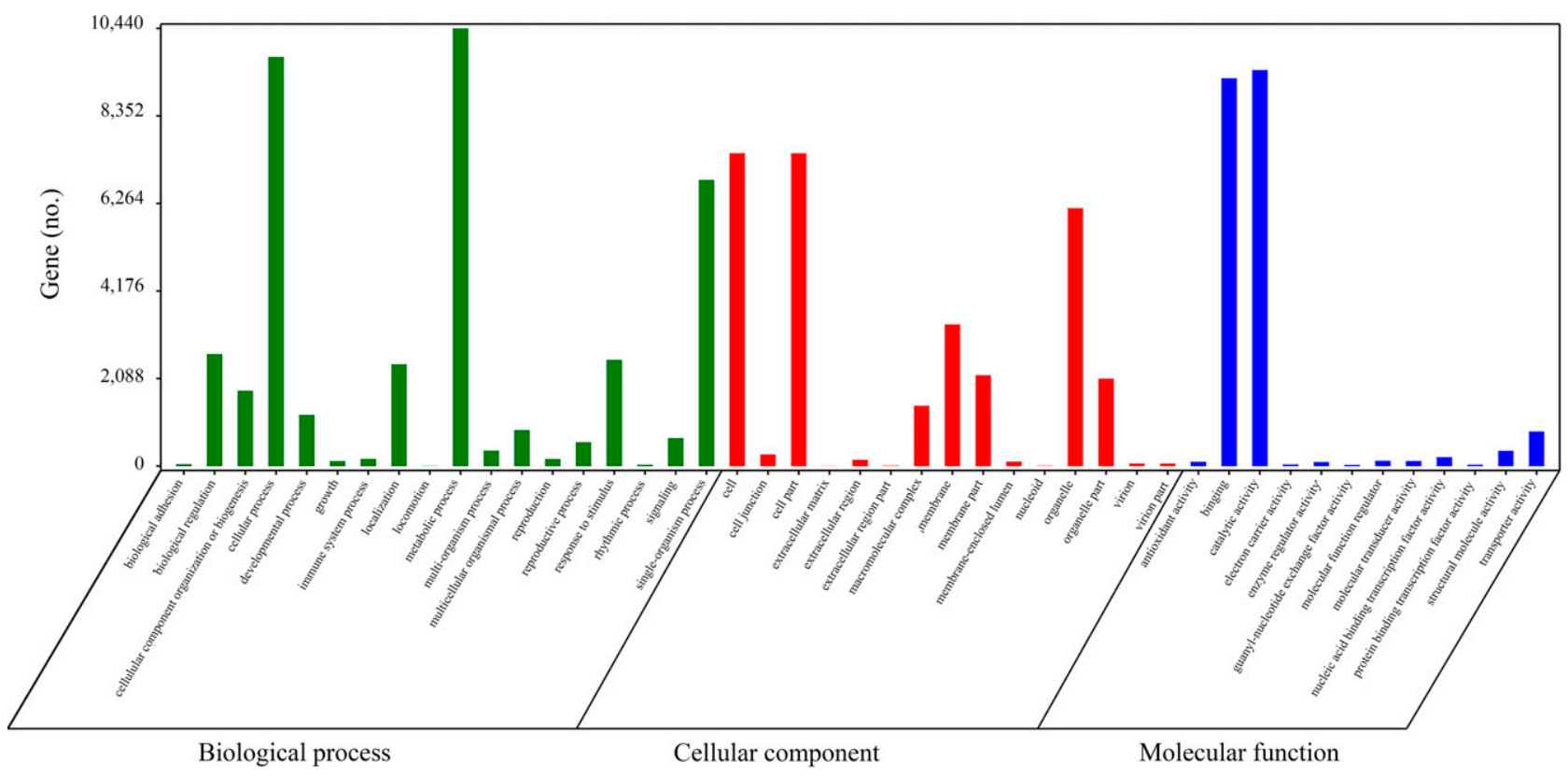

Fig. 6. Gene ontology (GO) classification of calla lily unigenes.

biosynthesis (upregulated). There were 842 DEGs annotated to 123 KEGG metabolic pathways in W vs. B. Among them, 31 were annotated to the phenylalanine pathway ( 23 upregulated and eight downregulated), 13 were annotated to the flavonoid biosynthesis pathway (11 upregulated and two downregulated), and three DEGs annotated to anthocyanin biosynthesis pathway (all downregulated), all of which were significantly enriched. In addition, one DEG was significantly related to flavonol biosynthesis. Among them, 32 (12 upregulated and 20 downregulated) DEGs were annotated to phenylalanine biosynthesis pathway, 11 (seven upregulated and four downregulated) DEGs were annotated to flavonoid biosynthesis pathway, four DEGs (all upregulated) were annotated to flavonoid biosynthesis pathway, and three DEGs (one upregulated and two downregulated) annotated. By means of DEG identification of anthocyanin biosynthesis pathway, the key genes of different colors of spathes were screened out and their coloring mechanism was analyzed. These DEGs laid a foundation for further research on the metabolism of flavonoids and related functional genes in the spathes of calla lily.

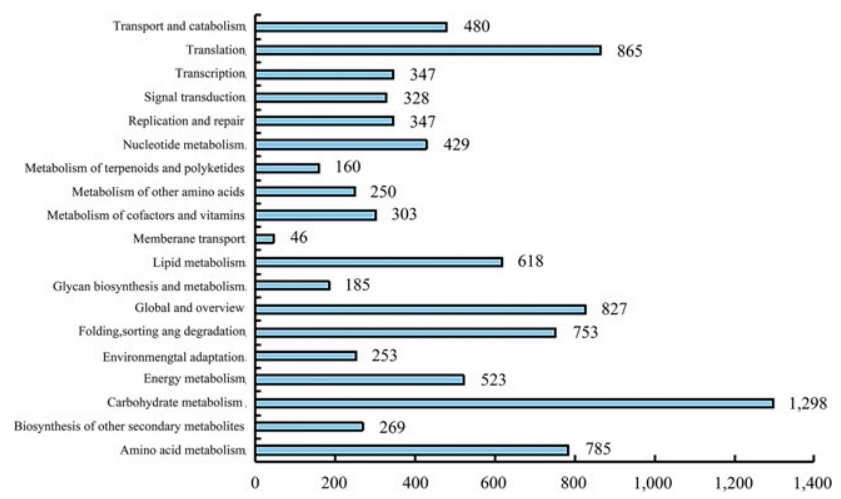

Fig. 7. Kyoto Encyclopedia of Genes and Genomes (KEGG) function classification of calla lily unigenes.
IDENTIFICATION OF DEGS AND KEY GENES RELATED TO THE ANTHOCYANIN BIOSYNTHESIS PATHWAY. To analyze the genes related to chromogenic substances in anthocyanin biosynthesis in the calla lily spathe [based on the RPKM values of Clematis florida (Kulahoglu et al., 2014)], 65 DEGs were screened to generate heatmaps (RPKM >30) and their $\log 2(\mathrm{RPKM})$ values were classified into three groups (Fig. 8, Supplemental Table 4). Cluster I was composed of 19 DEGs, namely CHS, CHI1, CHI2, DFR3, A3GT1, seven $M y b(M y b 2,5,8,9,11,13,14)$, four bHLH (bHLH2, 3, 12, 13), and three WD40 (WD40 7, 9, 10). The expression of these genes was downregulated in 'P,' and the structural gene $\mathrm{CHI} 2$ was the most downregulated (Fig. 8B Cluster I). There were 36 genes in Cluster II including $F 3 H, F 3^{\prime} H 1$, F3'H2, DFR1, DFR2, ANS, ANR1, ANR2, A3GT2, nine Myb (Myb1, 3, 4, 6, 7, 10, 12, 15, 16), six bHLH (bHLH1, 5, 6, 8-10), six WD40 (WD40 1-4, WD40 6, 8), and four GST (GST1, $3,7,8)$. The expression of structural genes in Cluster II was upregulated in $\mathrm{B}$ except that $D F R 2$ was significantly downregulated (Fig. 8B Cluster II). The Cluster III genes included two modifier genes $(A 3,5 G T 1,2)$, four transcription factors (bHLH4, 7, 11; WD40 5), and four transporters (GST2, 4-6) that were downregulated in 'B.'

Based on the functional annotation and hierarchical clustering of the $\log 2$ (RPKM) value, combined with the related map and DEG enrichment pathways identified by GO and KEGG, we screened the genes that may play a key role in the color of calla lily spathes. All these DEGs contained both upstream and downstream genes (Fig. 9). For example, compared with B, CHI2 transcriptional abundance was significantly downregulated (by $131 \%$ ) in $\mathrm{P}$, whereas $D F R 1$ and $D F R 2$ were significantly downregulated in $\mathrm{W}$ (by $126.5 \%$ and $560.8 \%$, respectively). The transcriptional abundance of $A N S, A N R 1$, and $A N R 2$ in $\mathrm{B}$ were significantly higher than in $\mathrm{W}$ by $312.3 \%, 327.9 \%$, and $624.6 \%$, respectively, and higher than in $\mathrm{P}$ by $52.1 \%, 347.6 \%$, and $728.8 \%$, respectively. The RPKM values of these six structural genes were significantly different among different cultivars. In 
A

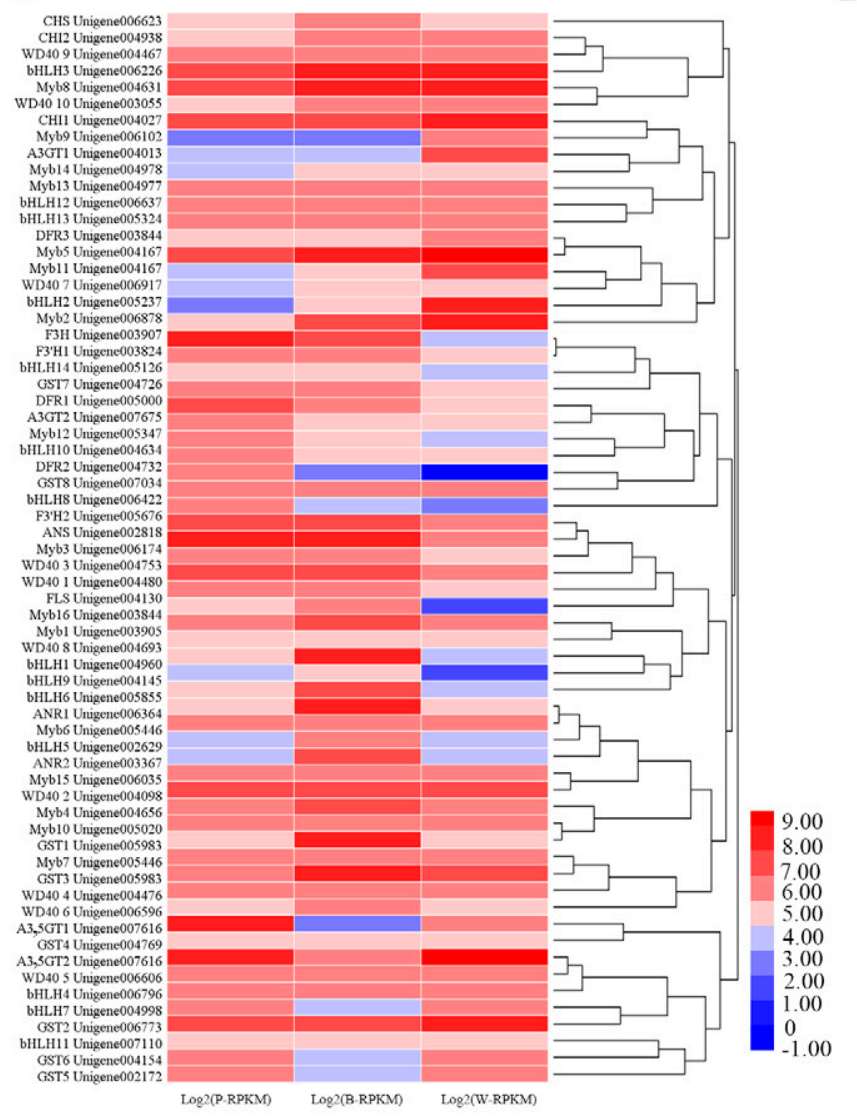

B

Cluster 1

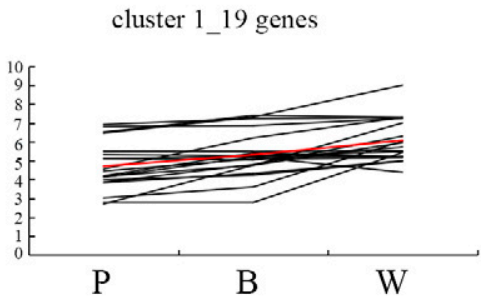

cluster 236 genes

Cluster 2

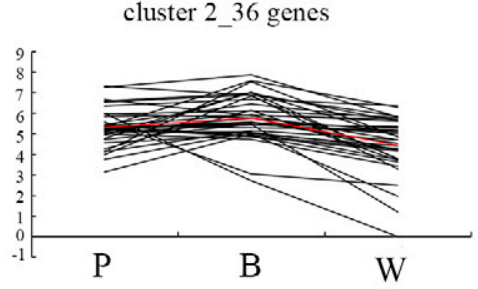

cluster 3_10 genes

Cluster 3

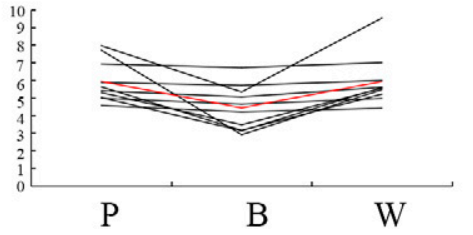

Fig. 8. (A) Expression profiles of anthocyanin biosynthetic genes differentially expressed in Romance (P), Black Girl (B), and Ventura (W) of calla lily cultivars. (B) Accumulation patterns of genes in three clusters. Data represent the log2 reads per kilobase per million (RPKM) mapped reads. The mean for each cluster is shown as a red line, and the expression patterns of individual genes are shown as gray lines.

addition, the transcription factor abundance of $b H L H 1$ in B was higher than in $\mathrm{W}$ and $\mathrm{P}$ by $856.6 \%$ and $371.3 \%$, respectively. The abundance of the transporter GST1 in B was higher than in $\mathrm{W}$ and $\mathrm{P}$ by $533.6 \%$ and $951.1 \%$, respectively.

VERIFICATION AND EXPRESSION ANALYSIS OF CANDIDATE GENES. The anthocyanin composition and content in the six differentcolored spathes were previously determined and analyzed. It was found that $\mathrm{W}$ and $\mathrm{Y}$ did not contain anthocyanins, $\mathrm{P}$ contained very few anthocyanins, and $\mathrm{B}, \mathrm{N}$, and $\mathrm{F}$ contained a large number of anthocyanins and flavonols, with $\mathrm{B}$ containing the most total anthocyanins, followed by N (X. Lei et al., 2017).

To verify the reproducibility and accuracy of the expression of DEGs identified by transcriptome analysis, we used qRT-PCR to determine the expression pattern of $\mathrm{P}, \mathrm{B}$, and $\mathrm{W}$. Using qRTPCR, we identified 11 structural genes $C H S, C H I 2, F 3 H, F 3^{\prime} H 1$, F3H'2, ANS, ANR1, ANR2, DFR 1, DFR 2, and FLS; one transporter GST1; and six transcription factors $M y b 2, M y b 5, b H L H 1$, bHLH5, bHLH6, and WD40 1. The expression patterns of CHS, CHI2, F3'H1, F3H'2, DFR1, DFR2, ANS, ANR1, ANR2, A3GT2, $M y b 2$, bHLH1, bHLH6, WD40 1, and GST1 were highly consistent with the results of the high-throughput sequencing (Fig. 10).

To further analyze the color mechanism of the calla lily spathe, the expression patterns of 19 genes in the six colored spathes $(\mathrm{P}, \mathrm{B}, \mathrm{W}, \mathrm{Y}, \mathrm{N}$, and $\mathrm{F})$ were analyzed showing that the expression of these 19 genes differed significantly among the different colors. These genes were highly expressed in $\mathrm{B}, \mathrm{N}$, and
$\mathrm{F}$ with high anthocyanin content but at very low levels in $\mathrm{W}, \mathrm{P}$, and $\mathrm{Y}$ that contained little or no anthocyanin. Analysis of the gene expression levels in $\mathrm{B}, \mathrm{N}$, and $\mathrm{F}$ showed that $A N R 1, A N R 2$, bHLH1, and GSTl were the most strongly expressed in B, whereas the expression levels of the other structural genes $A N S$, $C H S, F 3 H, F 3^{\prime} H s$, and DFRs were lower than in N. We found that the transcription factor $b H L H 1$ and transporter GST1 had RPKM values $>100$ in B and with a 5-fold difference in RPKM in W (which belongs to Cluster II), suggesting ANS may be regulated by bHLH1 to promote anthocyanin synthesis (Fig. 8). Correlation analysis showed that the bHLHI expression level was associated with increases in both the total anthocyanin content and ANS ( $R^{2}=0.995$ and 0.892 , respectively). Moreover, GST1 showed strong correlations with ANR1 and ANR2 $\left(R^{2}=\right.$ 0.997 and 0.997 , respectively). Interestingly, we also found that GST1 was highly correlated with the total anthocyanin content $\left(R^{2}=0.994\right)$. In addition, further analysis of the expression patterns of $\mathrm{W}, \mathrm{P}$, and $\mathrm{Y}$ showed the lowest expression level in the light colors, with trace expression of DFR 1 and DFR 2 in W but the highest expression of $\mathrm{CHI} 2 \mathrm{in} \mathrm{F}$.

\section{Discussion}

Overview. The biological regulation of flower color has attracted much attention in the study of ornamental plants (Lin et al., 2019). Anthocyanin is known to contribute to plant 


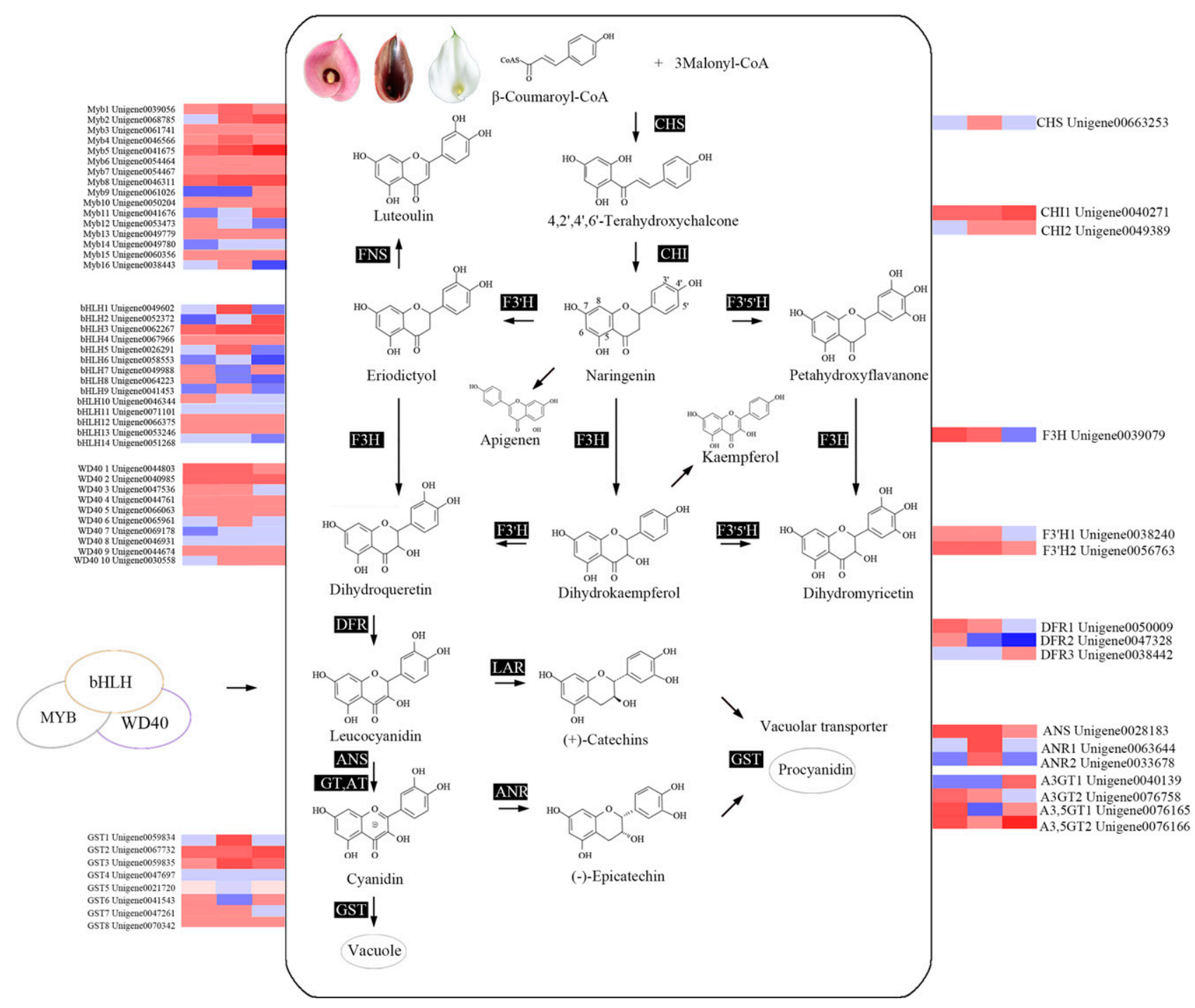

Fig. 9. Putative genes in the anthocyanin synthesis pathway and their expression levels. Heatmaps were constructed based on reads per kilobase per million mapped reads (RPKM) of pink, black, and white calla lily cultivars.

coloration (Rusishvili et al., 2019). In our study, we used spathes of the ornamental calla lily to identify the key genes underlying the color differences in the spathes and further analyzed the color mechanism. Taken together, our sequencing data provided an effective basis for the research of flower color gene resources for nonmodel plants.

Formation OF BLACK CALLA LILY SPATHES. Black flowers, fruits, leaves, seeds, and seedcoats are naturally found in various plant species (Markham et al., 2004). Previous studies on flower color development in the dahlia (Dahlia pinnata) suggest that a lack of flavonoids leads to a high accumulation of anthocyanins. Inhibition of $D v F N S$ expression eliminates the competition between flavonoid and anthocyanin synthesis, eventually leading to the accumulation of anthocyanins (Davies et al., 2003; Deguchi et al., 2015). In our previous study, we found a high accumulation of anthocyanins and flavonols in the spathes of B, and section microstructure showed that the pigment was widely distributed in the two to three layers of mesophyll cells in the upper and lower epidermis of B (X. Lei et al., 2017), leading to the formation of the black spathe.
ANS is a key enzyme in anthocyanin synthesis, which catalyzes the conversion of colorless anthocyanin into colored anthocyanin (Shi et al., 2018). For example, in sprenger's magnolia (Magnolia sprengeri) it was found that a higher transcription level of MSANS led to anthocyanin accumulation (Shi et al., 2014). It has been previously reported that the high ANS expression in black rice (Oryza sativa 'Hanzhong') results in the accumulation of anthocyanin, giving a purplish-black grain appearance of the caryopsis (Yin et al., 2017). According to the qRT-PCR results, the expression of $A N S$ in $\mathrm{B}$ was higher than in $\mathrm{N}$, possibly due to the dependency of the red spathe formation on anthocyanin accumulation, whereas the formation of black spathes may not only be caused by the accumulation of a large amount of anthocyanin.

Therefore, we speculated that there may be other mechanisms involved in the formation of the black spathe. Interestingly, the expression levels of $A N R 1$ and $A N R 2$ in the black spathe were the highest among the six cultivars. Previous studies have shown that $A N R$ is a key gene that catalyzes the transformation of anthocyanin into epicatechin ( $\mathrm{Li}$ et al., 2019). Therefore, we 

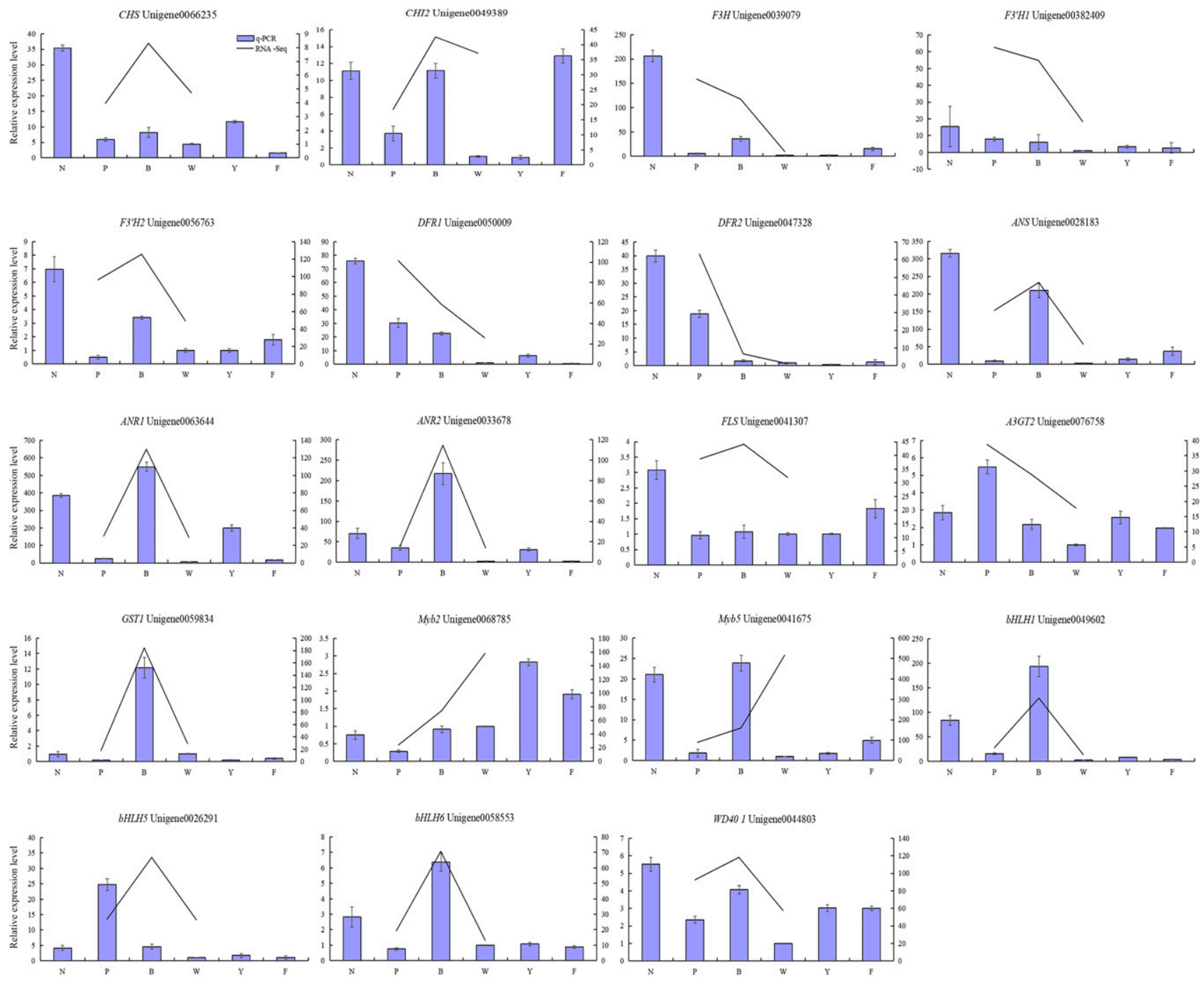

Fig. 10. Expression analysis of 19 genes related to anthocyanin biosynthesis in calla lilies using real-time quantitative polymerase chain reaction, including 12 structural genes (CHS, CHI2, F3H, F3'H1, F3'H2, DFR1, DFR2, ANS, ANR1, ANR2, FLS, and A3GT2), one transporter(GST1), and six transcription factors (Myb2, Myb5, bHLH1, bHLH5, bHLH6, and WD40 1). The Y-axis on the left represents the relative expression and on the right represents gene abundance; the X-axis depicts the six cultivars of calla lily, which are Black Girl (B), Romance (P), Ventura (W), Sun Club (Y), Promise (N), and Figo (F).

speculated that the high expression of $A N R 1$ and $A N R 2$ may lead to the accumulation of multiple procyanidins with possible interaction between anthocyanins and PAs, promoting the color deepening of the spathe. For example, experiments on the leaves of B, tobacco (Nicotiana tabacum), and Arabidopsis showed that there was an interaction between anthocyanin and PAs, and the interaction with PAs appeared to produce deeper color when procyanidin accumulation was high (Kitamura et al., 2004; Xie 2003). Large amounts of procyanidins and anthocyanins have been previously detected in the caryopses of black rice granules (Finocchiaro et al., 2010). These results support our hypothesis about the formation of the black spathe. In addition, the brown fibers of mature Arabidopsis and cotton (Gossypium hirsutum) may contain oxidized PAs (condensed tannins) and PA precursors (Debeaujon et al., 2001; Feng et al., 2013; Kitamura et al., 2004; Li et al., 2013; Tan et al., 2013; Xiao et al., 2007, 2014). Therefore, we believe that PA oxidation may also further promote the formation of the black spathe and we intend to investigate this in the future.
The INHIBITORY EFFECTS OF CHI AND DFR PLAY IMPORTANT ROLES IN THE FORMATION OF LIGHT-COLORED SPATHES IN THE CALLA LILY. This study suggested that the low expression of CHI2, DFR1, and DFR2 plays a key role in the formation of the pink or white spathe. Chalcone isomerase (CHI) is a stable enzyme involved in the early biosynthesis of anthocyanin, and promotes the molecular internalization of chalcone to form flavonoids (Nishihara et al., 2005; Wu et al., 2018). DFR catalyzes dihydroxyflavone alcohols (DHK, DHQ, and DHM) to colorless anthocyanins (LPG, LCY, and LDP) and has selective specificity for the three dihydroxyflavone alcohol substrates (Jin et al., 2016). The low expression of CHI2 in $\mathrm{P}$ may lead to the extremely low content of naringin it produces, so it rarely moves downstream to reduce the accumulation of anthocyanin. Meanwhile, the low expression of $F 3 H, D F R 1$, and $D F R 2$ leads to naringin development by the DHQ branch, leading to a low accumulation of cyanidin (Cy) so the spathe displays a light pink color. For example, inhibition of anthocyanin synthesis in transgenic tobacco may be related to the downregulation of $\mathrm{CHI}$ in 
the anthocyanin pathway (Han et al., 2012). Meanwhile, studies have shown that $\mathrm{Cy}$ is the main anthocyanin in pink peach (Prunus persica), and the expression of $C H I$ and $D F R$ in light-pink peach is lower than that in dark-pink peach. $C H I$ in the pink chrysanthemum (Chrysanthemum indicum) is also lower than in the white chrysanthemum (Chen et al., 2012b; Uematsu et al., 2012). In addition, our results showed high levels of CHI2 expression in $\mathrm{F}$, providing a large number of substrates and leading to the accumulation of $\mathrm{Cy}$, and the development of the red spathe color. Lower CHI2 expression, on the other hand, reduced the substrate availability, lowering the anthocyanin content, making the spathe lighter in color, and developing the above pink spathe in the process.

Anthocyanins were not detected in white or yellow calla lily spathes (X. Lei et al., 2017). This may be due to low expression of DFR 1 and DFR2 that affects anthocyanin synthesis, resulting in the extremely low anthocyanin content and the white spathe. According to existing studies, low expression of $F 3 H$ and $D F R$ in carnation (Dianthus caryophyllus) flowers leads to decreased anthocyanin accumulation, leading to the white flower phenotype, whereas $D F R$ is not expressed in the chrysanthemum white mutant (Feyissa et al., 2009; Totsuka et al., 2018). Thus, low $D F R$ expression may hinder anthocyanin synthesis, which is one of the causes of the whitening. Therefore, we hypothesized that the most likely reason for the formation of the white spathe in $\mathrm{W}$ was the extremely low expression levels of DFR1 and DFR2. As the anthocyanin synthesis pathway was inhibited and blocked, the synthesis of colorless anthocyanin was reduced, preventing anthocyanin synthesis and resulting in the light and white color.

THE FORMATION OF THE SPATHES MAY BE INFLUENCED BY TRANSCRIPTION FACTOR BHLH1 AND TRANSPORTER GST1 IN THE CALLA LILY. According to previous studies in other plants, $M y b, b H L H$, and $W D 40$ form MBW transcriptional activators that act upstream of the anthocyanin biosynthesis pathway genes (Dare et al., 2008). For example, experiments on tobacco have shown that $A N S$, regulated by $b H L H$ and $R 2 R 3-M y b$ transcription factors in flower color formation, can enhance the flower color of tobacco (Kim et al., 2018). The TT8 site of Arabidopsis encodes a $b H L H$ domain protein that regulates the biosynthesis of anthocyanins and procyanidins (Robbins et al., 2003). bHLH1 was consistently and highly correlated with anthocyanin content and $A N S$ expression in $\mathrm{B}$, indicating that $b H L H 1$ may promote anthocyanin synthesis by regulating ANS. The study on the coloration of different radish (Raphanus sativus) cultivars found that RsTT8 (bHLH, TF gene) was significantly positively correlated with the expression level of RsANS and other genes in the leaves and skins of the root flesh, promoting anthocyanin synthesis (Lim et al., 2017). In addition, a similar phenomenon was reported in the petals of Centaurea cyanus, which resulted in anthocyanin accumulation by CcbHLH1 (Deng et al., 2019). Moreover, the synthesis or accumulation of phytomelanin pigments in the seedcoat of Ipomoea purpurea was positively regulated by $b H L H 2$ (Park et al., 2007). Therefore, we speculate that bHLH1 may act as a positive regulator of the anthocyanin biosynthesis pathway, promoting anthocyanin synthesis and deepening the color of the spathe.

The coloration of plant floral organs is largely affected by the transport and accumulation of anthocyanins, and GST is one of the transporters responsible for the transport of anthocyanins. Meanwhile, some GSTs also participate in the transport of proanthocyanidins. For example, in Camelina sativa, CmGSTF12 is a functional GST that participates in both anthocyanin and PA accumulation (Wang et al., 2012). To date, although GST has been identified in many plant species, few of them have been identified as anthocyanin-related (Kitamura et al., 2012). The expression of GSTI in B was highly correlated with the anthocyanin content and the expression of $A N R 1$ and $A N R 2$. Similar studies have found that high expression of $C k m G S T 3$ plays an important role in the accumulation and transport of anthocyanin in cyclamen and peach, and the expression level of AcGST1 in Actinidia chinensis is consistent with the degree of anthocyanin accumulation (Cardoso et al., 2012; Cheng et al., 2015; Liu et al., 2019), whereas GhGSTF8 in the F family of cotton may be related to the transport and accumulation of anthocyanins or PAs (T. Lei et al., 2017). Therefore, we hypothesized that the proteins encoded by GSTI may be related to the accumulation and transport of both anthocyanins and PAs.

\section{Conclusions}

To analyze the effects of genes related to the anthocyanin biosynthesis pathway in the calla lily, the transcriptomic data of the $\mathrm{B}, \mathrm{P}$, and $\mathrm{W}$ cultivars were analyzed, and qRT-PCR was performed for six different-colored cultivars, including N, F, Y, together with the preceding three. The results showed that the formation of the black spathe is not only dependent on the accumulation of anthocyanins but also related to the interaction between numerous PAs. Meanwhile, bHLH1 may regulate anthocyanin synthesis, and GST1 is related to the transport of anthocyanins and PAs. However, the primary reason for the formation of the pink and white spathe is that low expression of CHI2, DFR1, and DFR2 leads to inhibition of the anthocyanin synthesis pathway. This study revealed the putative mechanism of color development in the spathes of the calla lily and provides effective genetic resources and a theoretical basis for flower color molecular breeding.

\section{Literature Cited}

Altschul, S.F., W. Gish, W. Miller, E.W. Myers, and D.J. Lipman. 1990. Basic local alignment search tool. Mol. Biol. 215:403-410, doi: 10.1006/jmbi.1990.9999.

Bueno, J.M., F. Ramos-Escudero, P. Sáez-Plaza, A.M. Muñoz, M.J. Navas, and A.G. Asuero. 2012. Analysis and antioxidant capacity of anthocyanin pigments. Part I: General considerations concerning polyphenols and flavonoids. Crit. Rev. Anal. Chem. 42(2):102-125, doi: 10.1080/10408347.2011.632312.

Cardoso, S., W. Lau, J.E. Dias, P. Fevereiro, and N. Maniatis. 2012. A candidate-gene association study for berry colour and anthocyanin content in Vitis vinifera L. PLoS One 7(9):e46021, doi: 10.1371/ journal.pone.0046021.

Chen, S.F., Y.Q. Zhou, Y.R. Chen, and J. Gu. 2018. Fastp: An ultrafast all-in-one FASTQ preprocessor. Bioinformatics 34(17):i884i890, doi: 10.1093/bioinformatics/bty560.

Chen, S.M., C.H. Li, X.R. Zhu, Y.M. Deng, W. Sun, L.S. Wang, F.D. Chen, and Z. Zhang. 2012a. The identification of flavonoids and the expression of genes of anthocyanin biosynthesis in the chrysanthemum flowers. Biol. Plant. 56(3):458-464, doi: 10.1007/s10535-0120069-3.

Chen, Q., H.W. Yu, H.R. Tang, and X.R. Wang. 2012b. Identification and expression analysis of genes involved in anthocyanin and proanthocyanidin biosynthesis in the fruit of blackberry. Scientia Hort. 141:61-68, doi: 10.1016/j.scienta.2012.04.025.

Cheng, J., L. Liao, H. Zhou, C. Gu, L. Wang, and Y.P. Han. 2015. A small indel mutation in an anthocyanin transporter causes variegated 
colouration of peach flowers. J. Expt. Bot. 66(22):7227-7239, doi: $10.1093 /$ jxb/erv419.

Cock, P.J.A., C.J. Fields, N. Goto, M.L. Heuer, and P.M. Rice. 2009. The Sanger FASTQ file format for sequences with quality scores, and the Solexa/Illumina FASTQ variants. Nucleic Acids Res. 38(6):1767-1771, doi: 10.1093/nar/gkp1137.

Consortium, U.P. 2020. UniProt: The universal protein knowledgebase in 2021. Nucleic Acids Res. 49(D1):D480-D489, doi: 10.1093/nar/ gkaa1100.

Dare, A.P., R.J. Schaffer, W.K. Lin, A.C. Allan, and R.P. Hellens. 2008. Identification of a cis-regulatory element by transient analysis of co-ordinately regulated genes. Plant Methods 4(1):1-10, doi: 10.1186/1746-4811-4-17.

Davies, K.M., K.E. Schwinn, S.C. Deroles, D.G. Manson, D.H. Lewis, S.J. Bloor, and J.M. Bradley. 2003. Enhancing anthocyanin production by altering competition for substrate between flavonol synthase and dihydroflavonol 4-reductase. Euphytica 131(3):259-268, doi: 10.1023/A:1024018729349.

Debeaujon, I., A.J.M. Peeters, K.M. Leon-Kloosterziel, and M. Koornneef. 2001. The TRANSPARENT TESTA 12 gene of Arabidopsis encodes a multidrug secondary transporter-like protein required for flavonoid sequestration in vacuoles of the seed coat endothelium. Plant Cell 13(4):853-871, doi: 10.1023/A:1024018729349.

Deguchi, A., F. Tatsuzawa, M. Hosokawa, M. Doi, and S. Ohno. 2015. Tobacco streak virus (strain dahlia) suppresses post-transcriptional gene silencing of flavone synthase II in black dahlia cultivars and causes a drastic flower color change. Planta 242(3):663-675, doi: 10.1007/s00425-015-2365-6.

Deng, C.Y., S.S. Li, C.Y. Feng, Y. Hong, H. Huang, J.Y. Wang, L.S. Wang, and S.L. Dai. 2019. Metabolite and gene expression analysis reveal the molecular mechanism for petal colour variation in six Centaurea cyanus cultivars. Plant Physiol. Biochem. 142:22-33, doi: 10.1016/j.plaphy.2019.06.018.

Feng, H.J., X.H. Tian, Y.C. Liu, Y.J. Li, X.Y. Zhang, B.J. Jones, Y.Q. Sun, and J. Sun. 2013. Analysis of flavonoids and the flavonoid structural genes in brown fiber of upland cotton. PLoS One 8(3):e58820, doi: 10.1371/journal.pone.0058820.

Feyissa, D.N., T. Løvdal, K.M. Olsen, R. Slimestad, and C. Lillo. 2009. The endogenous $G L 3$, but not $E G L 3$, gene is necessary for anthocyanin accumulation as induced by nitrogen depletion in Arabidopsis rosette stage leaves. Planta 230(4):747-754, doi: 10.1007/ s00425-009-0978-3.

Finocchiaro, F., B. Ferrari, and A. Gianinetti. 2010. A study of biodiversity of flavonoid content in the rice caryopsis evidencing simultaneous accumulation of anthocyanins and proanthocyanidins in a black-grained genotype. J. Cereal Sci. 51(1):28-34, doi: 10.1016/j. jcs.2009.09.003.

Francisco, R.M., A. Regalado, A. Ageorges, B.J. Burla, B. Bassin, C. Eisenach, O. Zarrouk, S. Vialet, T. Marlin, M.M. Chaves, E. Martinoia, and R. Nagy. 2013. ABCC1, an ATP binding cassette protein from grape berry, transports anthocyanidin 3-O-glucosides. Plant Cell 25(5): 1840-1854, doi:10.1105/tpc.112.102152.

Fu, X.P., D.C. Wang, X.L. Yin, P.C. Du, and B. Kan. 2014. Time course transcriptome changes in shewanella algae in response to salt stress. PLoS One 9(5):e96001, doi: 10.1371/journal.pone.0096001.

Grabherr, M.G., B.J. Haas, M. Yassour, J.Z. Levin, D.A. Thompson, I. Amit, X. Adiconis, L. Fan, R. Raychowdhury, Q.D. Zeng, Z.H. Chen, E. Mauceli, N. Hacohen, A. Gnirke, N. Rhind, F.D. Palma, B.W. Birren, C. Nusbaum, K. Lindblad-Toh, N. Friedman, and A. Regev. 2011. Fulllength transcriptome assembly from RNA-Seq data without a reference genome. Nat. Biotechnol. 29(7):644-652, doi: 10.1038/nbt.1883.

Grotewold, E. 2006. The genetics and biochemistry of floral pigments. Annu. Rev. Plant Biol. 57:761-780, doi: 10.1146/annurev.arplant.57. 032905.105248.

Guenter, S., M.A. Moseley, J. Sleep, and M. McGowran. 1998. The EMBL nucleotide sequence database. Nucl. Acids Res. 26(1):8-15, doi: 10.1093/nar/26.1.8.
Hanbali, L.B., Y.K. Nakhal, H.A. Hasan, R.M. Ghadieh, and J.J. Haddad. 2013. Measurement of antioxidant activity and antioxidant compounds under versatile extraction conditions: II. The immunobiochemical antioxidant properties of black sour cherry (Prunus cerasus) extracts. Anti-Inflammatory Anti-Allergy Agents Med. Chem. 12(3):229-245, doi: 10.2174/18715230113129990001.

Han, Y.P., S. Vimolmangkang, R.E. Soria-Guerra, and S.S. Korban. 2012. Introduction of apple $A N R$ genes into tobacco inhibits expression of both $C H I$ and $D F R$ genes in flowers, leading to loss of anthocyanin. Exp. Bot. 63(7):2437-2447, doi: 10.1093/jxb/err415.

Hu, L.S., C.Y. Hao, R. Fan, B.D. Wu, L.H. Tan, and H.S. Wu. 2015. De novo assembly and characterization of fruit transcriptome in black pepper (Piper nigrum). PLoS One 10(6):e0129822, doi: 10.1371/journal.pone.0129822.

Jin, X.H., H. Huang, L. Wang, Y. Sun, and S.L. Dai. 2016. Transcriptomics and metabolite analysis reveals the molecular mechanism of anthocyanin biosynthesis branch pathway in different Senecio cruentus cultivars. Front. Plant Sci. 7(107):1307, doi: 10.3389/fpls.2016. 01307.

Kelebek, H. and S. Selli. 2011. Evaluation of chemical constituents and antioxidant activity of sweet cherry (Prunus avium L.) cultivars. Int. J. Food Sci. Technol. 46(12):2530-2537, doi: 10.2174/18715230 11312999.0001.

Kim, D.H., S. Park, J.Y. Lee, S.H. Ha, and S.H. Lim. 2018. Enhancing flower color through simultaneous expression of the B-peru and $m P A P 1$ transcription factors under control of a flower-specific promoter. Int. J. Mol. Sci. 19(1):309-323, doi: 10.3390/ijms19010309.

Kitamura, S., N. Shikazono, and A. Tanaka. 2004. TRANSPARENT TESTA 19 is involved in the accumulation of both anthocyanins and proanthocyanidins in Arabidopsis. Int. J. Mol. Sci. 37(1):104-114, doi: 10.1046/j.1365-313x.2003.01943.x.

Kitamura, S., Y. Akita, H. Ishizaka, I. Narumi, and A. Tanaka. 2012. Molecular characterization of an anthocyanin-related glutathione Stransferase gene in cyclamen. J. Plant Physiol. 169(6):636-642, doi: 10.1016/j.jplph.2011.12.011.

Koes, R., W. Verweij, and F. Quattrocchio. 2005. Flavonoids: A colorful model for the regulation and evolution of biochemical pathways. Trends Plant Sci. 10(5):236-242, doi: 10.1016/j.tplants.2005.03.002.

Kulahoglu, C., A.K. Denton, M. Sommer, J. Maß, S. Schliesky, T.J. Wrobe, B. Berckmans, E. Gongora-Castillo, C.R. Buell, R. Simon, L.D. Veylder, A. Bräutigam, and A.P.M. Weber. 2014. Comparative transcriptome atlases reveal altered gene expression modules between two cleomaceae C3 and C4 plant species. Plant Cell 26(8):32433260, doi: 10.1105/tpc.114.123752.

Lei, T., Y. Song, X.H. Jin, T.Y. Su, and Y.W. Pu. 2017. Effects of pigment constituents and their distribution on spathe coloration of Zantedeschia hybrida. HortScience 52(12):1840-1848, doi: 10.21273/ hortsci12229-17.

Lei, X., C. Wen, S.Y. Guo, Y.Y. Huang, Y. Lin, Y.P. Cai, and J.S. Gao. 2017. Genome-wide analysis of the GST gene family in Gossypium hirsutum L. Hereditas 39(008):737-752, doi: 10.16288/j. yczz.16-435.

Lepiniec, L., I. Debeaujon, J.M. Routaboul, A. Baudry, L. Pourcel, N. Nesi, and M. Caboche. 2006. Genetics and biochemistry of seed flavonoids. Annu. Rev. Plant Biol. 57:405-430, doi: 10.1146/annurev. arplant.57.032905.105252.

Lewis, D.H., H.S. Arathoon, E.E. Swinny, S.C. Huang, and K.A. Funnell. 2003. Anthocyanin and carotenoid pigments in spathe tissue from selected Zantedeschia hybrids. Acta Hort. 624:147-154, doi: 10.17660/actahortic.2003.624.19.

Li, H., J. Tian, Y.Y. Yao, J. Zhang, T.T. Song, and K.T. Li. 2019. Identification of leucoanthocyanidin reductase and anthocyanidin reductase genes involved in proanthocyanidin biosynthesis in Malus crabapple plants. Plant Physiol. Biochem. 139:141-151, doi: 10.1016/j.plaphy.2019.03.003.

Li, M., Y.T. Cao, S.R. Ye, M. Irshad, T.F. Pan, and D.L. Qiu. 2016. Isolation of CHS gene from Brunfelsia acuminata flowers and its 
regulation in anthocyanin biosysthesis. Molecules 22(1):44, doi: 10.3390/Molecules22010044.

Li, P.H., L. Ponnala, N. Gandotra, L. Wang, Y.Q. Si, S.L. Tausta, T.H. Kebrom, N. Provart, R. Patel, and C.R. Myers. 2010. The developmental dynamics of the maize leaf transcriptome. Nat. Genet. 42(12):1060-1067, doi: 10.1038/ng.703.

Li, Y.J., X.Y. Zhang, F.X. Wang, C.L. Yang, F. Liu, G.X. Xia, and J. Sun. 2013. A comparative proteomic analysis provides insights into pigment biosynthesis in brown color fiber. J. Proteomics 78:374-388, doi: 10.1016/j.jprot.2012.10.005.

Lim, S.H., D.H. Kim, J.K. Kim, J.Y. Lee, and S.H. Ha. 2017. A radish basic helix-loop-helix tlranscription factor, RsTT8 acts a positive regulator for anthocyanin biosynthesis. Front. Plant Sci. 8:1917, doi: 10.3389/fpls.2017.01917.

Lin, Q.H., Q.Z. Zhong, and Z.H. Zhang. 2019. Comparative transcriptome analysis of genes involved in anthocyanin biosynthesis in the pink-white and red fruits of chinese bayberry (Morella rubra). Scientia Hort. 250:278-286, doi: 10.1016/j.scienta.2019.02.061.

Lin, Y., Y.H. Wang, B. Li, D.N. Li, L. Li, X. Liu, J.C. Han, and X.J. Meng. 2018. Comparative transcriptome analysis of genes involved in anthocyanin synthesis in blueberry. Plant Physiol. Biochem. 127:561-572, doi: 10.1016/j.plaphy.2018.04.034.

Liu, X.J., Y.N. Chuang, C.Y. Chiou, D.C. Chin, F.Q. Shen, and K.W. Yeh. 2012. Methylation effect on chalcone synthase gene expression determines anthocyanin pigmentation in floral tissues of two Oncidium orchid cultivars. Planta 236(2):401-409, doi: 10.1007/s00425012-1616-Z.

Liu, Y.F., Y.W. Qi, A.L. Zhang, H.X. Wu, Z.D. Liu, and X.L. Ren. 2019. Molecular cloning and functional characterization of AcGST1, an anthocyanin-related glutathione S-transferase gene in kiwifruit (Actinidia chinensis). Plant Mol. Biol. 100(4):1-15, doi: 10.1007/ s11103-019-00870-6.

Livak, K.J. and T.D. Schmittgen. 2001. Analysis of relative gene

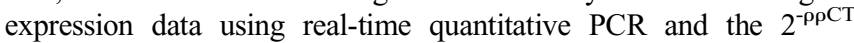
method. Methods 25(4):402-408, doi: 10.1006/meth.2001.1262.

Love, M.I., W. Huber, and S. Anders. 2014. Moderated estimation of fold change and dispersion for RNA-seq data with DESeq2. Genome Biol. 15(12):550, doi: 10.1186/s13059-014-0550-8.

Luo, H.F., C. Dai, Y.P. Li, J. Feng, Z.C. Liu, and C.Y. Kang. 2018. RAP codes for a GST anthocyanin transporter that is essential for the foliage and fruit coloration in strawberry. J. Expt. Bot. 69(10):2595-2608, doi: 10.1093/jxb/ery096.

Meng, X.Q., G. Li, L.Y. Gu, Y. Sun, Z.Y. Li, J.R. Liu, X.Q. Wu, T.T. Dong, and M.K. Zhu. 2019. Comparative metabolomic and transcriptome analysis reveal distinct flavonoid biosynthesis regulation between petals of white and purple Phalaenopsis amabilis. J. Plant Growth Regul. 39(3):823-840, doi: 10.1007/s00344-019-10025-y.

Markham, K.R., S.J. Bloor, R. Nicholson, R. Rivera, M. Shemluck, P.G. Kevan, and C. Michener. 2004. Black flower coloration in wild Lisianthius nigrescens: Its chemistry and ecological consequences. Z. Naturforsch. C 59(9-10):625-630, doi: 10.1515/znc-2004-9-1003.

Nguyen, D.T.T., D. Guillarme, S. Rudaz, and J.L. Veuthey. 2006. Chromatographic behaviour and comparison of column packed with sub- $2 \mu \mathrm{m}$ stationary phases in liquid chromatography. J. Chromatography 1128(1-2):105-113, doi: 10.1016/j.chroma.2006.06.069.

Nishihara, M., T. Nakatsuka, and S. Yamamura. 2005. Flavonoid components and flower color change in transgenic tobacco plants by suppression of chalcone isomerase gene. FEBS Lett. 579:6074-6078, doi: 10.1016/j.febslet.2005.09.073.

Nuala, A.O., M.W. Wright, J.R. Brister, S. Ciufo, D. Haddad, R. McVeigh, B. Rajput, B. Robbertse, B. Smith-White, D. Ako-Adjei, A. Astashyn, A. Badretdin, Y. Bao, O. Blinkova, V. Brover, V. Chetvernin, J. Choi, E. Cox, O. Ermolaeva, C.M. Farrell, T. Goldfarb, T. Gupta, D. Haft, E. Hatcher, W. Hlavina, V.S. Joardar, V.K. Kodali, W. Li, D. Maglott, P. Masterson, K.M. McGarvey, M.R. Murphy, K. O’Neill, S. Pujar, S.H. Rangwala, D. Rausch, L.D. Riddick, C. Schoch, A. Shkeda, S.S. Storz, H. Sun, F. Thibaud-Nissen, I. Tolstoy, R.E.
Tully, A.R. Vatsan, C. Wallin, D. Webb, W. Wu, M.J. Landrum, A. Kimchi, T. Tatusova, M. DiCuccio, P. Kitts, T.D. Murphy, and K.D. Pruitt. 2015. Reference sequence (RefSeq) database at NCBI: Current status, taxonomic expansion, and functional annotation. Nucleic Acids Res. 44(D1):D733-D745, doi: 10.1093/nar/gkv1189.

Nováková, L., L. Matysová, and P. Solich. 2006. Advantages of application of UPLC in pharmaceutical analysis. Talanta 68(3):908-918, doi: 10.1016/j.talanta.2005.06.035.

Ogata, H., S. Goto, K. Sato, W. Fujibuchi, H. Bono, and M. Kanehisa. 1999. KEGG: Kyoto encyclopedia of genes and genomes. Nucleic Acids Res. 1(1):29:34, doi: 10.1093/nar/27.1.29.

Ohmiya, A., 2010. Biosynthesis of plant pigments: Anthocyanins, betalains and carotenoids. Plant J. 54(4):733-749, doi: 10.1111/ j.1365-313X.2008.03447.x.

Park, K.I., N. Ishikawa, Y. Morita, J.D. Choi, A. Hoshino, and S. Iida. 2007. A $b H L H$ regulatory gene in the common morning glory, Ipomoea purpurea, controls anthocyanin biosynthesis in flowers, proanthocyanidin and phytomelanin pigmentation in seeds, and seed trichome formation. Plant J. 49(4):641-654, doi: 10.1111/j.1365-313x. 2006.02988.x.

Qu, Y., Z. Ou, F.S. Yang, S. Wang, and J.S. Peng. 2018. The study of transcriptome sequencing for flower coloration in different anthesis stages of alpine ornamental herb (Meconopsis 'Lingholm'). Gene 689:220-226, doi: 10.1016/j.gene.2018.12.017.

Ramsay, N.A. and B. Glover. 2005. MYB-bHLH-WD40 protein complex and the evolution of cellular diversity. Trends Plant Sci. 10(2):63-70, doi: 10.1016/j.tplants.2004.12.011.

Ricardo, P.D., M.E. José, S.C. Josselyn, G.V. Enrique, and R.L. Simón. 2016. Differential roles for VviGST1, VviGST3, and VviGST4 in proanthocyanidin and anthocyanin transport in Vitis vinifera. Front. Plant Sci. 7:1166, doi: 10.3389/fpls.2016.01166.

Robinson, M.D., D.J. McCarthy, and G.K. Smyth. 2009. edgeR: A bioconductor package for differential expression analysis of digital gene expression data. Bioinformatics 26(1):139-140, doi: 10.1093. bioinformatics/btp616.

Robbins, M.P., F. Paolocci, J.W. Hughes, V. Turchetti, G. Allison, S. Arcioni, P. Morris, and F. Damiani. 2003. Sn, a maize bHLH gene, modulates anthocyanin and condensed tannin pathways in Lotus corniculatus. J. Expt. Bot. 54(381):239-248, doi: 10.1093/jxb/erg022.

Rusishvili, M., L. Grisanti, S. Laporte, M. Micciarelli, M. Rosa, R.J. Robbins, T. Collins, A. Magistrato, and S. Baroni. 2019. Unraveling the molecular mechanisms of color expression in anthocyanins. Phys. Chem. Chem. Phys. 21:8757-8766, doi: 10.1039/c9cp00747d.

Saito, K., S.K. Yonekura, R. Nakabayashi, Y. Higashi, M. Yamazaki, T. Tohge, and A.R. Fernie. 2013. The flavonoid biosynthetic pathway in Arabidopsis: Structural and genetic diversity. Plant Physiol. Biochem. 72:21-34, doi: 10.1016/j.plaphy.2013.02.001.

Sheng, L.X., W. Xia, S. Zang, Y.Q. Zeng, X.Y. Yuan, G.G. Ning, S.C. Zhang, and L.G. Feng. 2018. Transcriptome-sequencing analyses reveal putative genes related to flower color variation in Chinese Rosa rugosa. Acta Physiol. Plant. 40(3):62, doi: 10.1007/s11738-018-2635-6.

Shi, L.Y., S.F. Cao, X. Chen, W. Chen, Y.H. Zeng, and Z.F. Yang. 2018. Proanthocyanidin synthesis in chinese bayberry (Myrica rubra Sieb. et Zucc.) fruits. Front. Plant Sci. 9:212, doi: 10.3389/ fpls.2018.00212.

Shi, S.G., S.J. Li, Y.X. Kang, and J.J. Liu. 2014. Molecular characterization and expression analyses of an anthocyanin synthase gene from Magnolia sprengeri Pamp. Appl. Biochem. Biotechnol. 175(1):477488, doi: 10.1007/s12010-014-1290-7.

Singh, N.V., T. Saminathan, R. Chandra, C. Awachare, K.D. Babu, D.M. Mundewadiker, and R.K. Pal. 2015. RNA isolation from high polyphenol containing tissues of pomegranate. Indian J. Hortic. 72(2):273-277, doi: 10.5958/0974-0112.2015.00051.1.

Singh, Y., A.E.V. Wyk, and H. Baijnath. 1996. Floral biology of Zantedeschia aethiopica (L.) Spreng. (Araceae). S. Afr. J. Bot. 62(3):146-150, doi: 10.1016/s0254-6299(15)30614-1. 
Tanaka, Y. and A. Ohmiya. 2008. Seeing is believing: Engineering anthocyanin and carotenoid biosynthetic pathways. Curr. Opin. Biotechnol. 19(2):190-197, doi: 10.1016/j.copbio.2008.02.015.

Tan, J.F., L.L. Tu, F.L. Deng, H.Y. Hu, Y.C. Nie, and X.L. Zhang. 2013. A genetic and metabolic analysis revealed that cotton fiber cell development was retarded by flavonoid naringenin. Plant Physiol. 162(1):86-95, doi: 10.1104/pp.112.212142.

Tatusov, R.L., N.D. Fedorova, J.D. Jackson, A.R. Jacobs, B. Kiryutin, E.V. Koonin, D.M. Krylov, R. Mazumder, S.L. Mekhedov, A.N. Nikolskaya, B.S. Rao, S. Smirnov, A.V. Sverdlov, S. Vasudevan, Y.I. Wolf, J.J. Yin, and D.A. Natale. 2003. The COG database: An updated version includes eukaryotes. BMC Bioinformatics 4(1):41, doi: 10.1186/1471-2105-4-41.

Totsuka, A., E. Okamoto, T. Miyahara, T. Kouno, E.A. Cano, N. Sasaki, A. Watanabe, K. Tasaki, M. Nishihara, and Y. Ozek. 2018. Repressed expression of a gene for a basic helix-loop-helix protein causes a white flower phenotype in carnation. Breed. Sci. 68(1):139-143, doi: 10.1270/jsbbs. 17072 .

Tripathi, A.M., A. Niranjan, and S. Roy. 2018. Global gene expression and pigment analysis of two contrasting flower color cultivars of Canna. Plant Physiol. Biochem. 127:1-10, doi: 10.1016/j.plaphy. 2018.03.008

Uematsu C., K. Uozumi, I. Makino, O. Arakawa, H. Katayama, C. Martin. 2012. Flower color variegation in single petal flowering peach. Acta Hort. 929(929):287-292, doi: 10.17660/actahortic.2012.929.42

Wang, Y., Y. Tang, M. Zhang, F. Cai, X. Li, J. Qin, Q. Wang, C. Liu, G. Wang, L. Xu, L. Yang, J. Li, Z. Wang, and X. Li. 2012. Molecular cloning and functional characterization of a glutathione S-transferase involved in both anthocyanin and proanthocyanidin accumulation in Camelina sativa (Brassicaceae). Genet. Mol. Res. 11(4):4711-4719, doi: 10.4238/2012.September.25.4.

Wei, Z.Z., Z.Z. Sun, B.B. Cui, Q.X. Zhang, M. Xiong, X. Wang, and D. Zhou. 2016. Transcriptome analysis of colored calla lily (Zantedeschia rehmannii Engl.) by Illumina sequencing: De novo assembly, annotation and EST-SSR marker development. PeerJ 4(9):e2378, doi: 10.7717 /peerj.2378.

Wu, Y.Q., L. Liu, D.Q. Zhao, and J. Tao. 2018. Age-associated methylation change of CHI promoter in herbaceous peony (Paeonia lactiflora Pall). Biosci. Rep. 38(5):BSR20180482, doi: 10.1042/ BSR2018048.2.

Xiao, Y.H., Q. Yan, H. Ding, M. Luo, L. Hou, M. Zhang, D. Yao, H.S. Liu, X. Li, J. Zhao, and Y. Pei. 2014. Transcriptome and biochemical analyses revealed a detailed proanthocyanidin biosynthesis pathway in brown cotton fiber. PLoS One 9(1):e86344, doi: 10.1371/ journal.pone.0086344.

Xiao, Y.H., Z.S. Zhang, M.H. Yin, M. Luo, X.B. Li, L. Hou, and Y. Pei. 2007. Cotton flavonoid structural genes related to the pigmentation in brown fibers. Biochem. Biophys. Res. Commun. 358(1):73-78, doi: 10.1016/j.bbrc.2007.04.084.

Xie, D.Y. 2003. Role of anthocyanidin reductase, encoded by BANYULS in plant flavonoid biosynthesis. Science 299(5605):396399, doi: 10.1126/science. 1078540.

Xu, W.J., C. Dubos, and L. Lepiniec. 2015. Transcriptional control of flavonoid biosynthesis by MYB-bHLH-WDR complexes. Trends Plant Sci. 20(3):176-185, doi: 10.1016/j.tplants.2014.12.001.

Yin, Y.J., T. Zhang, L. Wang, H.Z. Lu, and W.L. Du. 2017. Cloning and bioinformatic analysis of anthocyanidin synthase gene from Hanzhong black rice. Fujian. J. Agr. Sci. 32(002):124-129, doi: 10.19303/j.issn.1008-0384.2017.02.444.

Zheng, J., Z.H. Hu, X.L. Guan, D.Q. Dou, G. Bai, Y. Wang, Y.T. Guo, W. Li, and P.S. Leng. 2015. Transcriptome analysis of Syringa oblata Lindl. Inflorescence identifies genes associated with pigment biosynthesis and scent metabolism. PLoS One 10(11):e0142542, doi: 10.1371/journal.pone. 0142542 .

Zhou, C.B., X. Mei, D.O. Rothenberg, Z.B. Yang, W.T. Zhang, S.H. Wan, H.J. Yang, and L.Y. Zhang. 2020, Metabolome and transcriptome analysis reveals putative genes involved in anthocyanin accumulation and coloration in white and pink tea (Camellia sinensis) flower. Molecules 25(1):190, doi: 10.3390/molecules.25010190. 
Supplemental Table 1. Potential flavenoid/anthocyanin biosynthesis pathway genes examined by real-time quantitative PCR (qRT-PCR).

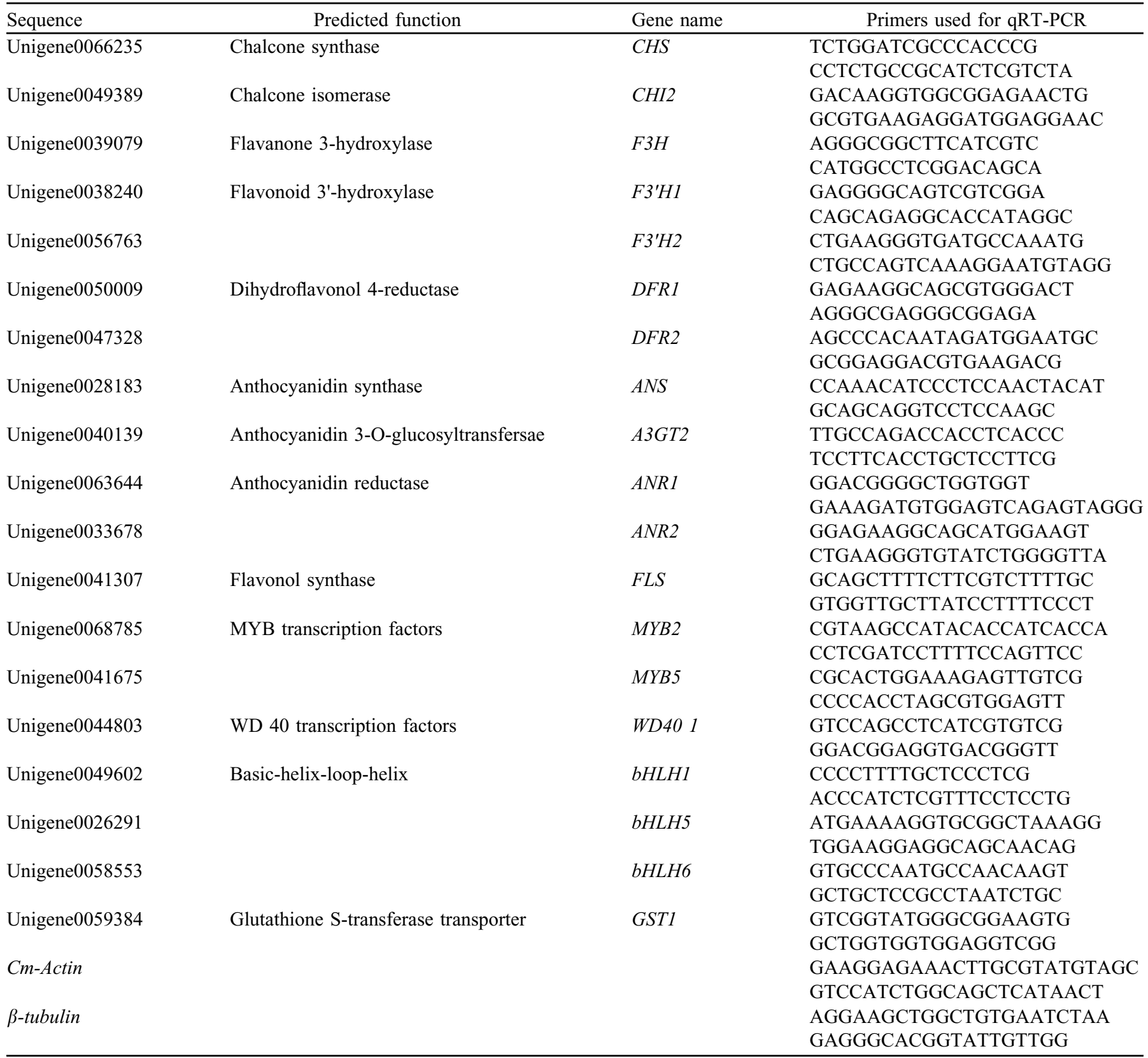


Supplemental Table 2. Significantly enriched gene ontology (GO) in calla lily of different colors $(P<0.05)$.

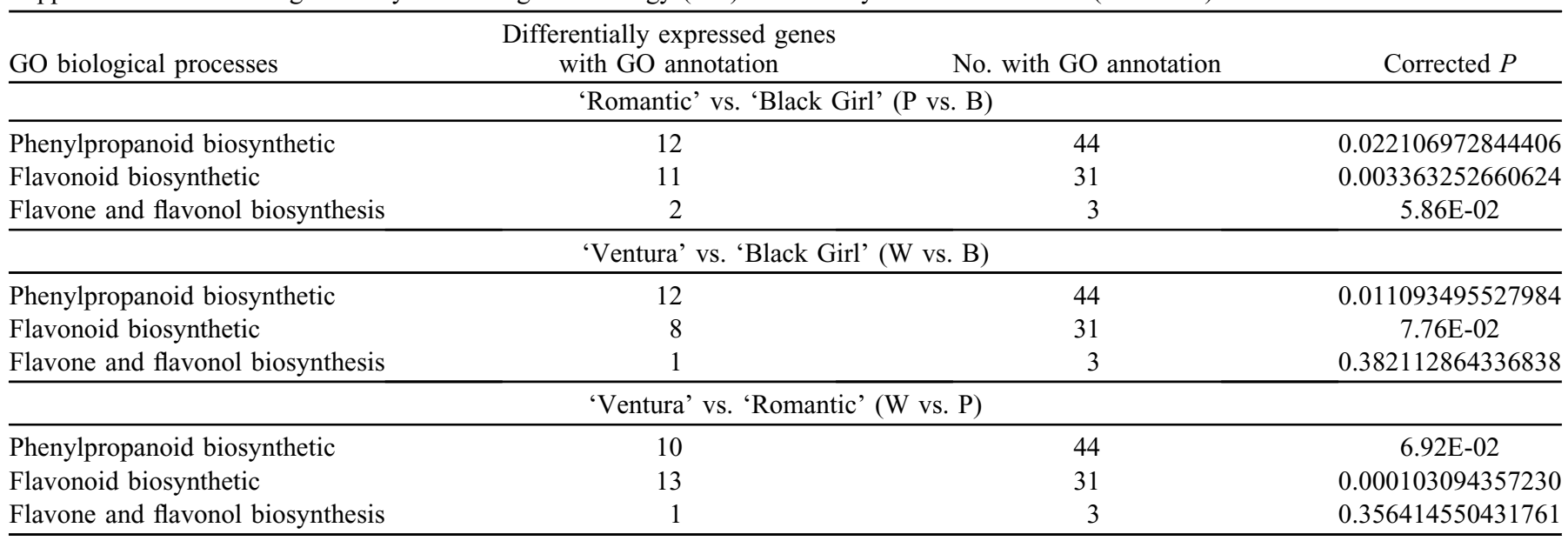

Supplemental Table 3. Kyoto Encyclopedia of Genes and Genomes (KEGG) classification of calla lily.

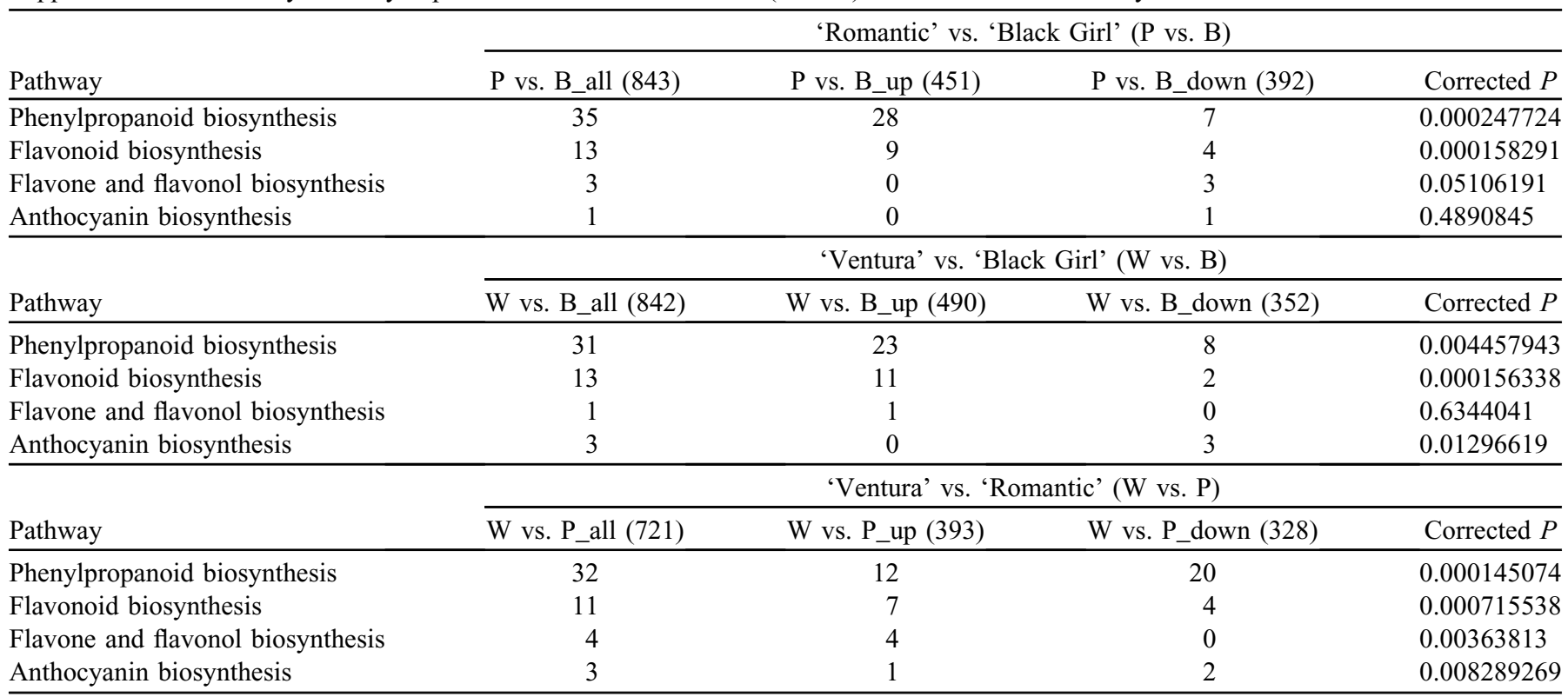

Significantly enriched KEGG biosynthesis of other secondary metabolites in calla lily of different colors $(P<0.05)$. 
Supplemental Table 4. Filter cluster graph data of reads per kilobase per million mapped reads (RPKM) in 'Romantic' (P), 'Black girl' (B), and 'Ventura' (W) of calla lily.

\begin{tabular}{|c|c|c|c|c|c|c|c|}
\hline Gene No. & Gene Name & P_rpkm & $\log 2(\mathrm{rpkm})$ & B_rpkm & $\log 2(\mathrm{rpkm})$ & W_rpkm & $\log 2(\mathrm{rpkm})$ \\
\hline$\overline{C H S}$ & Unigene0066235 & 17.5252 & 4.131359003 & 36.8624 & 5.204078098 & 20.8831 & 4.384263984 \\
\hline $\mathrm{CHII}$ & Unigene 0040271 & 112.7844 & 6.817423722 & 116.0702 & 6.85885381 & 152.073 & 7.24862022 \\
\hline CHI2 & Unigene0049389 & 18.4015 & 4.201751467 & 42.5071 & 5.409631931 & 37.1303 & 5.214525067 \\
\hline $\mathrm{F} 3 \mathrm{H}$ & Unigene 0039079 & 158.3587 & 7.307052319 & 117.0474 & 6.870949078 & 9.691 & 3.276645543 \\
\hline$F 3^{\prime} H 1$ & Unigene 0038240 & 62.5006 & 5.965798134 & 54.7305 & 5.774273131 & 18.2316 & 4.188369272 \\
\hline$F 3^{\prime} H 2$ & Unigene 0056763 & 96.0365 & 6.585510921 & 125.3603 & 6.969936727 & 49.0454 & 5.616045926 \\
\hline DFR1 & Unigene0050009 & 101.2303 & 6.661497368 & 58.4637 & 5.869469231 & 25.8132 & 4.690037095 \\
\hline$D F R 2$ & Unigene0047328 & 63.0861 & 5.979250261 & 6.5262 & 2.706243201 & 0.9876 & -0.018001258 \\
\hline DFR3 & Unigene 0038442 & 17.9615 & 4.166835932 & 27.0418 & 4.757119281 & 62.1207 & 5.957002182 \\
\hline$A N S$ & Unigene 0028183 & 152.7179 & 7.254725359 & 232.2287 & 7.859402469 & 56.3211 & 5.815603606 \\
\hline$A 3 G T 2$ & Unigene 0040139 & 8.1634 & 3.02917015 & 12.2006 & 3.608880193 & 65.3877 & 6.030947373 \\
\hline$A N R 1$ & Unigene 0063644 & 30.3812 & 4.92510695 & 129.6899 & 7.018922319 & 28.9715 & 4.856562477 \\
\hline ANR2 & Unigene 0033678 & 15.7607 & 3.978259707 & 114.1955 & 6.835361991 & 13.7773 & 3.784221279 \\
\hline$F L S$ & Unigene 0041307 & 38.6458 & 5.2722397 & 43.981 & 5.458808502 & 31.8907 & 4.99506386 \\
\hline$A 3,5 G T 1$ & Unigene0076165 & 208.6819 & 7.705161664 & 7.4892 & 2.904811618 & 44.0166 & 5.459975805 \\
\hline$A 3,5 G T 2$ & Unigene0076166 & 249.1895 & 7.961099469 & 40.285 & 5.33217085 & 739.9972 & 9.531376002 \\
\hline Mybl & Unigene 0039056 & 61.7618 & 5.948642894 & 68.8724 & 6.105854046 & 55.2925 & 5.789011898 \\
\hline Myb2 & Unigene 0068785 & 23.6116 & 4.561423902 & 73.998 & 6.209414373 & 157.7955 & 7.301912253 \\
\hline Myb3 & Unigene 0061741 & 57.1779 & 5.837385729 & 59.4531 & 5.893680132 & 52.1185 & 5.703723658 \\
\hline Myb4 & Unigene 0046566 & 43.5255 & 5.443788966 & 86.7113 & 6.438148109 & 51.4631 & 5.685466459 \\
\hline Myb5 & Unigene 0041675 & 88.2529 & 6.463571781 & 159.0144 & 7.313013609 & 514.1869 & 9.006149045 \\
\hline Myb6 & Unigene0054464 & 35.0956 & 5.133218263 & 53.5372 & 5.742469783 & 34.6468 & 5.114650205 \\
\hline$M y b 7$ & Unigene0054467 & 41.5874 & 5.378074587 & 60.5883 & 5.920967322 & 48.6475 & 5.604293762 \\
\hline$M y b 8$ & Unigene0046311 & 121.3644 & 6.923201486 & 150.7524 & 7.23603716 & 155.3291 & 7.279184325 \\
\hline$M y b 9$ & Unigene0061026 & 6.9045 & 2.787536943 & 6.8872 & 2.783917573 & 43.2012 & 5.432999482 \\
\hline Myblo & Unigene 0050204 & 37.4206 & 5.225760786 & 46.1895 & 5.529493024 & 39.1834 & 5.292170683 \\
\hline Mybl1 & Unigene 0041676 & 14.5169 & 3.859661502 & 27.7812 & 4.796037012 & 78.8755 & 6.30150534 \\
\hline Myb12 & Unigene 0053473 & 42.4136 & 5.406455037 & 29.3386 & 4.874728124 & 12.315 & 3.622344723 \\
\hline Mybl3 & Unigene0049779 & 34.9249 & 5.12618408 & 32.9338 & 5.041497079 & 52.9179 & 5.725683906 \\
\hline Mybl4 & Unigene 0049780 & 15.8726 & 3.988466562 & 18.8256 & 4.234623941 & 31.3838 & 4.971948142 \\
\hline Myb15 & Unigene 0060356 & 33.4553 & 5.064162876 & 43.454 & 5.44141708 & 33.869 & 5.081893486 \\
\hline$b H L H 1$ & Unigene0049602 & 27.2957 & 4.77060179 & 128.6541 & 7.007353624 & 13.4484 & 3.749362636 \\
\hline bHLH2 & Unigene0049603 & 6.5445 & 2.710282974 & 27.0483 & 4.757466018 & 128.4032 & 7.004537347 \\
\hline bHLH3 & Unigene0049604 & 91.8605 & 6.521372731 & 169.0636 & 7.401422266 & 158.1652 & 7.305288398 \\
\hline bHLH4 & Unigene0049605 & 58.7047 & 5.875404108 & 52.1139 & 5.703596319 & 63.3708 & 5.985746323 \\
\hline bHLH5 & Unigene0049606 & 13.4229 & 3.746624492 & 33.5016 & 5.066158094 & 13.2529 & 3.72823618 \\
\hline bHLH6 & Unigene 0049607 & 19.111 & 4.256331365 & 70.5515 & 6.140604851 & 12.9943 & 3.699807013 \\
\hline bHLH7 & Unigene 0049608 & 32.347 & 5.015560012 & 8.7365 & 3.127055426 & 47.9405 & 5.583173051 \\
\hline bHLH8 & Unigene0049609 & 47.3886 & 5.566468135 & 8.2063 & 3.036731896 & 5.5589 & 2.474799429 \\
\hline bHLH9 & Unigene 0049610 & 8.7531 & 3.129794053 & 31.5075 & 4.977623381 & 3.866 & 1.950841638 \\
\hline bHLH10 & Unigene0049611 & 36.6734 & 5.19666212 & 31.9309 & 4.996881312 & 21.0728 & 4.397310117 \\
\hline bHLH11 & Unigene0049612 & 31.4025 & 4.972807514 & 24.9426 & 4.640539953 & 31.1335 & 4.960395867 \\
\hline bHLH12 & Unigene0049613 & 45.2021 & 5.498317894 & 44.887 & 5.488225773 & 46.4064 & 5.536251879 \\
\hline bHLH13 & Unigene0049614 & 39.946 & 5.319979141 & 38.4409 & 5.264570209 & 44.3967 & 5.47238054 \\
\hline bHLH14 & Unigene0049615 & 31.8628 & 4.993801147 & 26.2918 & 4.716541011 & 10.6842 & 3.417406982 \\
\hline WD40 1 & Unigene 0044803 & 92.2956 & 6.528189967 & 118.0325 & 6.883040347 & 57.3774 & 5.84241069 \\
\hline WD40 2 & Unigene 0044804 & 81.5793 & 6.350131223 & 101.2037 & 6.661118226 & 82.2297 & 6.36158766 \\
\hline WD40 3 & Unigene 0044805 & 39.1044 & 5.289259043 & 47.4618 & 5.568694911 & 22.9891 & 4.522878082 \\
\hline WD40 4 & Unigene0044806 & 38.9902 & 5.28503965 & 43.316 & 5.436828119 & 40.5885 & 5.342999119 \\
\hline WD40 5 & Unigene 0044807 & 42.1601 & 5.397806384 & 33.0766 & 5.04773904 & 48.5813 & 5.602329191 \\
\hline$W D 406$ & Unigene 0044808 & 17.4808 & 4.127699305 & 35.5021 & 5.14983246 & 25.9042 & 4.695114124 \\
\hline WD40 7 & Unigene0044809 & 14.2809 & 3.836014998 & 20.055 & 4.325890061 & 31.9667 & 4.998497914 \\
\hline WD40 8 & Unigene 0044810 & 23.4084 & 4.548954422 & 30.3126 & 4.921845696 & 18.7159 & 4.22619252 \\
\hline WD40 9 & Unigene0044811 & 34.9079 & 5.125481664 & 37.5051 & 5.229014884 & 37.1382 & 5.214831988 \\
\hline WD40 10 & Unigene 0044812 & 21.8324 & 4.448398827 & 34.2471 & 5.097909923 & 35.7299 & 5.159059971 \\
\hline GST1 & Unigene0059834 & 17.4865 & 4.128169651 & 183.8009 & 7.522000021 & 29.0071 & 4.858334163 \\
\hline
\end{tabular}


Supplemental Table 4. (Continued) Filter cluster graph data of reads per kilobase per million mapped reads (RPKM) in 'Romantic' (P), 'Black girl' (B), and 'Ventura' (W) of calla lily.

\begin{tabular}{|c|c|c|c|c|c|c|c|}
\hline Gene No. & Gene Name & P_rpkm & $\log 2(\mathrm{rpkm})$ & B_rpkm & $\log 2(\mathrm{rpkm})$ & W_rpkm & $\log 2(\mathrm{rpkm})$ \\
\hline$\overline{G S T 2}$ & Unigene0067732 & 121.2352 & 6.921664828 & 105.099 & 6.715605132 & 128.1919 & 7.002161296 \\
\hline GST3 & Unigene 0059835 & 39.9164 & 5.318909707 & 190.6335 & 7.574657856 & 77.8184 & 6.282039413 \\
\hline GST4 & Unigene 0047697 & 23.607 & 4.561142809 & 18.3615 & 4.198612016 & 21.2513 & 4.409479192 \\
\hline GST5 & Unigene 0021720 & 39.3798 & 5.299383879 & 11.045 & 3.465321514 & 46.473 & 5.538320874 \\
\hline GST6 & Unigene 0041543 & 49.3833 & 5.625951342 & 8.8692 & 3.14880398 & 36.9224 & 5.206424428 \\
\hline GST7 & Unigene0047261 & 33.8453 & 5.0808836 & 33.4209 & 5.06267868 & 19.2249 & 4.264904189 \\
\hline GST8 & Unigene0070342 & 41.4751 & 5.374173555 & 39.0238 & 5.286282364 & 36.7147 & 5.198285906 \\
\hline
\end{tabular}

